\title{
Year in Review 2007
}

\section{Year in review: communicable disease surveillance, NSW, 2007}

\section{Communicable Diseases Branch, NSW Department of Health}

In this issue, we present our annual review of notifiable diseases among New South Wales (NSW) residents. Readers interested in the details of notifications for specific diseases are referred to in Tables 2-6 where diseases are reported by: year of onset; month of onset; Area Health Service (AHS); and age group and sex.

\section{Trends}

Among the 46706 notifications of medical conditions by doctors, hospital staff and laboratory staff in NSW residents in 2007, highlights included:

\section{Conditions most frequently reported}

- Chlamydia trachomatis infections: 12447 cases (181 per 100000 population) with the highest crude rates by geographical area in the South Eastern Sydney Illawarra (Randwick region), Sydney South West (Camperdown region), Hunter New England (Tamworth region) and Greater West (Broken Hill region) AHSs.

- Hepatitis C: 4259 cases (62 per 100000 population) with the highest crude rates in the Greater Western (Broken Hill region), North Coast (Lismore region) and Sydney South West (Camperdown region) AHSs.

- Hepatitis B: 2656 cases (39 per 100000 population) with the highest crude rates in the Sydney South West (Camperdown and Liverpool regions) and Sydney West (Parramatta region) AHSs.

- Salmonella infections: 2564 cases (37 per 100000 population) with the highest crude rates in the North Coast (Lismore region), Northern Sydney Central Coast (Gosford region) and Sydney South West (Camperdown region) AHSs.

- Pertussis: 2093 cases (30 per 100000 population) with the highest crude rates in Greater Western (Dubbo region), Sydney West (Parramatta region) and South Eastern Sydney Illawarra (Randwick region) AHSs.
Conditions with the most meaningful declines in the number of notifications compared with previous years

- Measles: four cases in 2007, the lowest annual count to date and a striking decrease compared with 2348 cases notified in 1993. No local measles transmission occurred in 2007 with all four cases resulting from exposure overseas.

- Meningococcal serogroup C disease: 10 cases reported for 2007, the lowest number of notifications since laboratory reporting began in 1991, largely due to the introduction of meningococcal $\mathrm{C}$ vaccination in late 2003.

- Gonorrhoea: 1384 cases in 2007 compared with 1736 cases in 2006, a decrease of $20 \%$.

- Hepatitis A: a record low number of 65 cases, decreased from 1119 in 1991, perhaps in part due to the introduction of a commercially based vaccination in the 1990s. Travel to endemic countries was the most commonly reported risk factor for disease acquisition in 2007.

- Psittacosis: 34 cases, a 64\% decrease compared with 2006.

- Leptospirosis: eight cases, down from 66 in 2001.

Conditions with the most meaningful increases in the number of notifications compared with previous years

- Salmonella infections: 2564 cases, the highest annual count to date. ${ }^{1}$ This increase is mainly due to a large point-source outbreak affecting 319 people who ate Vietnamese-style pork or chicken rolls from a bakery.

- Infectious syphilis, primarily affecting homosexual men residing in metropolitan Sydney.

- Mumps: 323 cases, a steady increase from 28 cases in 2001. This increase occurred mainly in the second half of the year in young adults in South East Sydney Illawarra (Randwick region) AHS. 
- Legionnaire disease: 73 reported Legionella pneumophila infections in part due to an outbreak in Sydney central business district in January.

- Influenza with high rates of disease reported in July and August. There were 25 reported influenza (influenza A) outbreaks in 2007: 21 in aged-care

Table 1. The five most commonly reported notifiable diseases by age group, NSW, 2007

\begin{tabular}{|c|c|}
\hline Age group & Rate/100000 \\
\hline \multicolumn{2}{|l|}{ Children under 5 years } \\
\hline 1. Salmonella infection & 146 \\
\hline 2. Giardiasis & 129 \\
\hline 3. Influenza & 97 \\
\hline 4. Cryptosporidiosis & 44 \\
\hline 5. Pertussis & 41 \\
\hline \multicolumn{2}{|l|}{ Children (5 to 15 years) } \\
\hline 1. Salmonella infection & 37 \\
\hline 2. Giardiasis & 27 \\
\hline 3. Chlamydia*\# & 24 \\
\hline 4. Pertussis & 21 \\
\hline 5. Influenza & 19 \\
\hline \multicolumn{2}{|l|}{ Young adults (16 to 24 years) } \\
\hline 1. Chlamydia* & 818 \\
\hline 2. Hepatitis C & 53 \\
\hline 3. Hepatitis B & 48 \\
\hline 4. Gonorrhoea & 40 \\
\hline 5. Salmonella infection & 40 \\
\hline \multicolumn{2}{|l|}{ Adults ( 25 to 44 years) } \\
\hline 1. Chlamydia* & 241 \\
\hline 2. Hepatitis C & 124 \\
\hline 3. Hepatitis B & 74 \\
\hline 4. Gonorrhoea & 41 \\
\hline 5. Giardiasis & 32 \\
\hline \multicolumn{2}{|l|}{ Adults (45 to 64 years) } \\
\hline 1. Hepatitis C & 70 \\
\hline 2. Pertussis & 39 \\
\hline 3. Hepatitis B & 37 \\
\hline 4. Arboviral infection & 34 \\
\hline 5. Chlamydia* & 30 \\
\hline \multicolumn{2}{|l|}{ Older Adults (65 years) } \\
\hline 1. Influenza & 36 \\
\hline 2. Pertussis & 33 \\
\hline 3. Salmonella infection & 24 \\
\hline 4. Arboviral infection & 21 \\
\hline 5. Invasive pneumococcal disease & 19 \\
\hline \multicolumn{2}{|c|}{ * refers to Chlamydia trachomatis infection. } \\
\hline \multicolumn{2}{|c|}{$\begin{array}{l}\text { \# two-thirds of the notifications reported in this age group were in } \\
15 \text { year olds. Where a case is reported in a child under16 years } \\
\text { old, the relevant public health unit contacts the treating doctor } \\
\text { outlining his/her obligation to notify the Department of } \\
\text { Community Services. }\end{array}$} \\
\hline \multicolumn{2}{|l|}{ Source: NSW Notifiable Diseases Database. } \\
\hline
\end{tabular}

facilities, three in military facilities and one in a boarding school.

- Verotoxigenic Escherichia coli (VTEC) infections: 23 cases reported, compared with 10 cases reported in 2006. All cases were investigated and no epidemiological links were identified.

- Giardiasis: 1940 cases reported compared with 1725 reported in 2006.

\section{Conditions least frequently reported}

There were no reported cases of anthrax, avian influenza, botulism, chancroid, diphtheria, granuloma inguinale, lyssavirus, plague, polio, rabies, severe acute respiratory syndrome (SARS), smallpox, tularaemia, typhus, viral haemorrhagic fever or yellow fever in NSW in 2007.

\section{Top five notifiable diseases}

Rates for the most commonly reported notifiable diseases for each age group and geographical area of residence at the time of notification are presented in Fig. 1 and Table 1. These lists indicate the relative importance of notifiable diseases only and should not be used to indicate the spread of all infectious diseases in NSW. It should also be noted that these rates are heavily influenced by testing practices and, in many instances, do not necessarily indicate the true or relative incidence in the community. Finally, these lists do not include the institutional gastrointestinal outbreaks as comprehensive demographic data are not collected for such outbreaks.

\section{Geographical distribution of notifiable diseases}

- Chlamydia trachomatis infection was the most commonly reported infection across NSW with highest rates observed in regional areas followed by rural and metropolitan areas.

- Rates of hepatitis $\mathrm{C}$ infection were comparable across rural, regional and metropolitan areas. Most of these cases will represent chronic infection rather than acute hepatitis $\mathrm{C}$ acquisition and as such may not accurately reflect the recent spread of the hepatitis $\mathrm{C}$ epidemic.

- Arboviral infections are more commonly reported in people residing in rural and regional areas than in metropolitan areas, relating to the distribution of infected mosquitoes.

- Higher rates of disease are reported for Justice Health compared with the rest of NSW, likely related to higher testing rates for bloodborne viruses and sexually transmitted infections on entry into correctional facilities. Within this population, hepatitis $\mathrm{C}$ was the most commonly reported infection, attributable to high rates of injecting drug use.

\section{Age distribution of notifiable diseases}

- Gastrointestinal and respiratory diseases are most commonly reported in children aged under 5 years. This is influenced by the higher testing rates in this age group. 
Table 2. Disease notifications by year of onset of illness a, NSW, 1991-2007

\begin{tabular}{|c|c|c|c|c|c|c|c|c|c|c|c|c|c|c|c|c|c|}
\hline Condition & 1991 & 1992 & 1993 & 1994 & 1995 & 1996 & 1997 & 1998 & 1999 & 2000 & 2001 & 2002 & 2003 & 2004 & 2005 & 2006 & 2007 \\
\hline Adverse event after immunisation & 9 & 31 & 23 & 40 & 28 & 56 & 70 & 95 & 16 & 42 & 111 & 178 & 219 & 186 & 107 & 70 & 224 \\
\hline Anthrax & 0 & 0 & 0 & 0 & 0 & 0 & 0 & 0 & 0 & 0 & 0 & 0 & 0 & 0 & 0 & 1 & 0 \\
\hline Arboviral infection & 408 & 343 & 656 & 381 & 539 & 1227 & 1806 & 783 & 1220 & 980 & 1191 & 665 & 1024 & 1148 & 1088 & 1917 & 1498 \\
\hline Barmah Forest virus ${ }^{b}$ & 6 & 6 & 25 & 39 & 271 & 172 & 185 & 134 & 249 & 197 & 401 & 396 & 451 & 403 & 448 & 644 & 573 \\
\hline Ross River virus ${ }^{b}$ & 297 & 324 & 599 & 331 & 236 & 1031 & 1598 & 583 & 952 & 750 & 717 & 183 & 494 & 701 & 584 & 1221 & 841 \\
\hline Other ${ }^{b}$ & 105 & 13 & 32 & 11 & 32 & 24 & 23 & 66 & 19 & 33 & 73 & 86 & 79 & 44 & 56 & 52 & 84 \\
\hline Blood lead level $\geq 15 \mathrm{ug} / \mathrm{dL}^{\mathrm{b}}$ & \multicolumn{5}{|c|}{ Not notifiable until December 1996} & & 710 & 874 & 691 & 985 & 513 & 516 & 338 & 304 & 234 & 298 & 263 \\
\hline Botulism & 0 & 0 & 0 & 0 & 0 & 0 & 0 & 0 & 1 & 0 & 0 & 0 & 0 & 1 & 0 & 0 & 0 \\
\hline Brucellosis $^{b}$ & 2 & 2 & 4 & 4 & 2 & 1 & 3 & 3 & 2 & 1 & 1 & 2 & 3 & 7 & 3 & 10 & 3 \\
\hline Chancroid $^{b}$ & Not no & tifiable & until De & cembe & 1998 & & & & 1 & 0 & 0 & 0 & 0 & 0 & 0 & 0 & 0 \\
\hline Chlamydia trachomatis infection & & & & & & & & & 2469 & 3509 & 4500 & 5823 & 7788 & 10030 & 11285 & 12057 & 12447 \\
\hline Congenital chlamydiab & Not no & tifiable & until Au & gust 19 & & & & & 14 & 18 & 16 & 15 & 23 & 28 & 46 & 39 & 31 \\
\hline Chlamydia - other ${ }^{\mathrm{b}}$ & Not no & tifiable & until Au & gust 19 & & & & & 2455 & 3491 & 4484 & 5808 & 7765 & 10002 & 11239 & 12018 & 12416 \\
\hline Cholera $^{\mathrm{b}}$ & 1 & 0 & 1 & 0 & 1 & 3 & 1 & 1 & 2 & 0 & 1 & 1 & 0 & 1 & 0 & 3 & 2 \\
\hline Creutzfeldt-Jakob disease $^{b}$ & Not no & tifiable & until $\mathrm{Ap}$ & ril 2004 & & & & & & & & & & 6 & 8 & 10 & 7 \\
\hline Cryptosporidiosis $^{b}$ & Not no & tifiable & until $\mathrm{De}$ & cembe & 1996 & & 157 & 1130 & 121 & 134 & 195 & 306 & 203 & 357 & 849 & 778 & 544 \\
\hline Foodborne illness (NOS)e & 2765 & 253 & 106 & 213 & 270 & 211 & 255 & 201 & 151 & 147 & 56 & 41 & 1071 & 550 & 309 & 507 & 763 \\
\hline Gastroenteritis (institutional) & 158 & 406 & 443 & 296 & 1359 & 554 & 939 & 738 & 673 & 697 & 775 & 1752 & 3583 & 12784 & 1395 & 10641 & 10488 \\
\hline Giardiasis $^{b}$ & Not no & tifiable & until $A u$ & gust 19 & & & & & 1091 & 978 & 967 & 863 & 1028 & 1234 & 1448 & 1725 & 1940 \\
\hline Gonorrhoeab $^{b}$ & 392 & 491 & 382 & 357 & 428 & 522 & 636 & 1054 & 1291 & 1060 & 1364 & 1527 & 1328 & 1442 & 1579 & 1736 & 1384 \\
\hline Haemolytic uraemic syndrome & Not no & tifiable & until De & cember & 1996 & & 3 & 6 & 11 & 9 & 2 & 7 & 5 & 9 & 11 & 11 & 13 \\
\hline Haemophilus influenzae serotype ${ }^{b}$ & 212 & 217 & 124 & 61 & 29 & 13 & 17 & 11 & 13 & 8 & 7 & 10 & 6 & 5 & 7 & 11 & 7 \\
\hline Hib epiglottitis ${ }^{b}$ & 15 & 57 & 32 & 21 & 6 & 2 & 5 & 1 & 2 & 2 & 1 & 1 & 0 & 3 & 0 & 1 & 1 \\
\hline Hib meningitis ${ }^{b}$ & 48 & 103 & 53 & 17 & 11 & 4 & 3 & 3 & 3 & 1 & 1 & 1 & 0 & 0 & 2 & 0 & 2 \\
\hline Hib septi & 11 & 26 & 24 & 12 & 8 & 3 & 1 & 4 & 6 & 4 & 2 & 3 & 1 & 2 & 4 & 6 & 2 \\
\hline Hib infection NOSb & 138 & 31 & 15 & 11 & 4 & 4 & 8 & 3 & 2 & 1 & 3 & 5 & 5 & 0 & 1 & 4 & 2 \\
\hline Hepatitis $A^{b}$ & 1119 & 901 & 579 & 585 & 614 & 958 & 1426 & 927 & 421 & 201 & 197 & 149 & 124 & 137 & 83 & 95 & 65 \\
\hline Hepatitis B & 1492 & 3169 & 3603 & 3983 & 4007 & 3504 & 3167 & 2957 & 3508 & 3972 & 4556 & 3546 & 2845 & 2812 & 2742 & 2518 & 2656 \\
\hline Hepatitis B - acute viral & 409 & 112 & 95 & 74 & 61 & 43 & 53 & 58 & 77 & 100 & 94 & 88 & 74 & 53 & 56 & 53 & 56 \\
\hline Hepatitis B - other ${ }^{b}$ & 1083 & 3057 & 3508 & 3909 & 3946 & 3461 & 3114 & 2899 & 3431 & 3872 & 4462 & 3458 & 2771 & 2759 & 2686 & 2465 & 2600 \\
\hline Hepatitis C & 851 & 3895 & 5896 & 7820 & 6878 & 7000 & 6926 & 7206 & 8598 & 8297 & 8654 & 6694 & 5249 & 4915 & 4364 & 4392 & 4259 \\
\hline Hepatitis C - acute viral ${ }^{b}$ & 22 & 26 & 22 & 16 & 32 & 18 & 19 & 112 & 112 & 222 & 295 & 152 & 127 & 59 & 43 & 55 & 53 \\
\hline Hepatitis C - other ${ }^{b}$ & 829 & 3869 & 5874 & 7804 & 6846 & 6982 & 6907 & 7094 & 8486 & 8075 & 8359 & 6542 & 5122 & 4856 & 4321 & 4337 & 4206 \\
\hline Hepatitis $D^{b}$ & 0 & 8 & 12 & 19 & 19 & 9 & 11 & 3 & 14 & 12 & 11 & 9 & 12 & 14 & 15 & 15 & 11 \\
\hline Hepatitis $E^{b}$ & 0 & 0 & 1 & 2 & 0 & 3 & 6 & 4 & 7 & 9 & 6 & 6 & 6 & 8 & 7 & 10 & 8 \\
\hline HIV infection ${ }^{b}$ & 823 & 693 & 589 & 502 & 536 & 447 & 423 & 402 & 377 & 352 & 340 & 393 & 413 & 407 & 391 & 369 & 404 \\
\hline Influenza & Not no & tifiable & until De & cember & 2000 & & & & & & 244 & 1012 & 861 & 1011 & 1414 & 617 & 1918 \\
\hline Influenza - Type $A^{b}$ & Not no & tifiable & until De & cember & 2000 & & & & & & 216 & 770 & 767 & 797 & 1055 & 421 & 1487 \\
\hline Influenza - Type $B^{b}$ & Not no & tifiable & until De & cember & 2000 & & & & & & 27 & 241 & 55 & 161 & 280 & 150 & 180 \\
\hline Influenza - Type A \& B & Not no & tifiable & until De & cember & 2003 & & & & & & & & & 26 & 65 & 37 & 43 \\
\hline Influenza - Type NOS ${ }^{b}$ & Not no & tifiable & until De & cember & 2000 & & & & & & 1 & 1 & 39 & 27 & 14 & 9 & 208 \\
\hline Legionellosis & 37 & 104 & 66 & 60 & 75 & 74 & 33 & 46 & 41 & 41 & 68 & 44 & 60 & 80 & 89 & 78 & 105 \\
\hline Legionella longbeachae $^{b}$ & 0 & 14 & 13 & 8 & 16 & 30 & 9 & 19 & 12 & 12 & 29 & 21 & 37 & 27 & 24 & 22 & 29 \\
\hline L.pneumophilab & 16 & 80 & 34 & 30 & 35 & 34 & 18 & 22 & 22 & 26 & 38 & 22 & 23 & 51 & 64 & 55 & 73 \\
\hline Legionnaire disease other & 21 & 10 & 19 & 22 & 24 & 10 & 6 & 5 & 7 & 3 & 1 & 1 & 0 & 2 & 1 & 1 & 3 \\
\hline Leprosy & 1 & 7 & 5 & 3 & 3 & 2 & 0 & 0 & 1 & 2 & 4 & 0 & 2 & 5 & 1 & 1 & 4 \\
\hline Leptospirosis $^{b}$ & 28 & 21 & 16 & 14 & 6 & 33 & 33 & 50 & 56 & 54 & 66 & 39 & 39 & 40 & 35 & 18 & 8 \\
\hline Listeriosis $^{b}$ & 11 & 13 & 12 & 10 & 14 & 22 & 23 & 28 & 22 & 18 & 12 & 11 & 28 & 30 & 25 & 26 & 22 \\
\hline Lymphogranuloma venereum (LGV) ${ }^{\mathrm{b}}$ & 0 & 0 & 0 & 0 & 0 & 0 & 0 & 0 & 0 & 0 & 0 & 0 & 0 & 1 & 2 & 1 & 0 \\
\hline Malariab $^{\mathrm{b}}$ & 171 & 110 & 174 & 184 & 96 & 204 & 173 & 158 & 174 & 232 & 157 & 105 & 120 & 101 & 206 & 140 & 98 \\
\hline Measles & 495 & 805 & 2348 & 1484 & 596 & 191 & 273 & 119 & 32 & 36 & 31 & 8 & 18 & 12 & 5 & 60 & 4 \\
\hline Measles laboratory confirmed & 19 & 76 & 460 & 302 & 138 & 35 & 98 & 19 & 13 & 22 & 18 & 6 & 14 & 11 & 4 & 48 & 4 \\
\hline Measles - other & 476 & 729 & 1888 & 1182 & 458 & 156 & 175 & 100 & 19 & 14 & 13 & 2 & 4 & 1 & 1 & 12 & 0 \\
\hline Meningococcal disease & 128 & 121 & 153 & 142 & 113 & 161 & 218 & 186 & 221 & 253 & 234 & 216 & 202 & 149 & 140 & 107 & 112 \\
\hline Meningococcal - serogroup $\mathrm{B}^{\mathrm{b}}$ & 0 & 3 & 7 & 7 & 23 & 36 & 53 & 55 & 95 & 93 & 90 & 105 & 100 & 81 & 73 & 54 & 76 \\
\hline Meningococcal - serogroup $\mathrm{C}^{\mathrm{b}}$ & 0 & 4 & 6 & 9 & 8 & 35 & 55 & 55 & 60 & 64 & 38 & 54 & 45 & 24 & 16 & 13 & 10 \\
\hline Meningococcal - serogroup W135b & 0 & 0 & 0 & 0 & 1 & 0 & 2 & 4 & 4 & 4 & 2 & 2 & 2 & 5 & 8 & 5 & 2 \\
\hline Meningococcal - serogroup $\mathrm{Y}^{\mathrm{b}}$ & 0 & 0 & 1 & 1 & 0 & 1 & 0 & 7 & 1 & 7 & 2 & 2 & 5 & 3 & 3 & 1 & 5 \\
\hline Meningococcal - other & 128 & 114 & 139 & 125 & 81 & 89 & 108 & 65 & 61 & 85 & 102 & 53 & 50 & 36 & 40 & 34 & 19 \\
\hline Mumps $^{b}$ & 8 & 23 & 13 & 11 & 14 & 27 & 29 & 39 & 33 & 92 & 28 & 29 & 35 & 65 & 111 & 155 & 323 \\
\hline Paratyphoidd,d & 20 & 8 & 9 & 11 & 12 & 15 & 5 & 9 & 5 & 14 & 11 & 13 & 22 & 10 & 0 & 0 & 0 \\
\hline Pertussis & 49 & 217 & 1533 & 1405 & 1369 & 1156 & 4246 & 2309 & 1415 & 3691 & 4437 & 2012 & 2772 & 3569 & 5809 & 4918 & 2093 \\
\hline Pneumococcal disease (invasive) ${ }^{b}$ & Not no & tifiable & until De & cember & 2000 & & & & & & 444 & 862 & 802 & 906 & 641 & 565 & 522 \\
\hline Psittacosis ${ }^{b}$ & Not no & tifiable & until De & cember & 2000 & & & & & & 38 & 155 & 87 & 81 & 121 & 94 & 34 \\
\hline Q fever ${ }^{\mathrm{b}}$ & 167 & 213 & 403 & 267 & 201 & 287 & 258 & 236 & 164 & 132 & 144 & 310 & 288 & 223 & 143 & 175 & 215 \\
\hline Rubella & 60 & 324 & 1186 & 233 & 2376 & 636 & 153 & 78 & 46 & 191 & 58 & 35 & 24 & 18 & 10 & 37 & 9 \\
\hline Congenital rubella ${ }^{b}$ & 1 & 0 & 2 & 4 & 1 & 5 & 0 & 0 & 1 & 0 & 0 & 0 & 1 & 1 & 0 & 0 & 1 \\
\hline Rubella - other ${ }^{b}$ & 59 & 324 & 1184 & 229 & 2375 & 631 & 153 & 78 & 45 & 191 & 58 & 35 & 23 & 17 & 10 & 37 & 8 \\
\hline Salmonella infection ${ }^{b, d}$ & 1115 & 819 & 1001 & 1125 & 1393 & 1250 & 1721 & 1826 & 1470 & 1426 & 1671 & 2112 & 1842 & 2145 & 2184 & 2071 & 2564 \\
\hline Shigellosis ${ }^{b}$ & Not no & tifiable & until De & cember & 2000 & & & & & & 134 & 85 & 59 & 96 & 135 & 75 & 71 \\
\hline Syphilis & 580 & 873 & 730 & 963 & 835 & 662 & 510 & 611 & 584 & 580 & 547 & 646 & 842 & 1042 & 841 & 892 & 1115 \\
\hline Congenital syphilis & 1 & 1 & 0 & 2 & 6 & 3 & 3 & 0 & 3 & 2 & 1 & 1 & 3 & 1 & 5 & 4 & 4 \\
\hline Infectious syphilis b,c & 1 & 3 & 6 & 29 & 132 & 72 & 57 & 45 & 86 & 81 & 67 & 128 & 244 & 302 & 242 & 232 & 434 \\
\hline Syphilis - other ${ }^{b}$ & 578 & 869 & 724 & 932 & 697 & 587 & 450 & 566 & 495 & 497 & 479 & 517 & 595 & 739 & 594 & 656 & 677 \\
\hline Tetanus & 5 & 2 & 5 & 4 & 0 & 1 & 3 & 3 & 1 & 3 & 0 & 0 & 1 & 1 & 1 & 2 & 2 \\
\hline Tuberculosis ${ }^{b}$ & 429 & 394 & 389 & 394 & 443 & 410 & 422 & 382 & 483 & 448 & 416 & 447 & 386 & 431 & 452 & 463 & 452 \\
\hline Typhoid $^{b}$ & 11 & 3 & 7 & 1 & 0 & 3 & 5 & 1 & 0 & 3 & 5 & 14 & 13 & 35 & 25 & 31 & 26 \\
\hline $\begin{array}{l}\text { Verotoxin-producing } \\
\text { Escherichia coli infections }{ }^{b}\end{array}$ & Not no & tifiable & I De & aber & 96 & & 0 & 2 & 0 & 1 & 1 & 6 & 3 & 5 & 16 & 10 & 23 \\
\hline
\end{tabular}

aYear of onset: the earlier of patient reported onset date, specimen date or date of notification. 'bLaboratory-confirmed cases only. Includes Syphilis primary, Syphilis secondary, Syphilis < 1 year duration and Syphilis newly acquired. 'DFrom 2005, all paratyphoid recorded as salmonellosis. eFoodborne illness cases are only those notified as part of an outbreak.

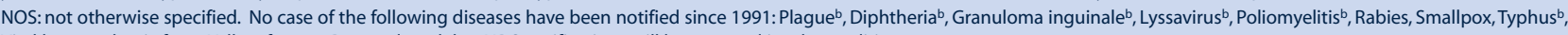
Viral haemorrhagic fever, Yellow fever. Due to data delay AIDS notifications will be reported in a later edition. 
Table 3. Disease notifications by month of onset of illness a , NSW, 2007

\begin{tabular}{|c|c|c|c|c|c|c|c|c|c|c|c|c|c|}
\hline Conditions & Jan. & Feb. & Mar. & Apr. & May & June & July & Aug. & Sept. & Oct. & Nov. & Dec. & Total \\
\hline Adverse event after immunisation & 6 & 3 & 11 & 11 & 59 & 39 & 20 & 23 & 21 & 13 & 13 & 5 & 224 \\
\hline Anthrax & 0 & 0 & 0 & 0 & 0 & 0 & 0 & 0 & 0 & 0 & 0 & 0 & 0 \\
\hline Arboviral infection & 97 & 97 & 163 & 234 & 196 & 99 & 83 & 80 & 89 & 114 & 127 & 119 & 1498 \\
\hline Barmah Forest virus ${ }^{b}$ & 43 & 35 & 76 & 125 & 77 & 29 & 32 & 27 & 27 & 34 & 38 & 30 & 573 \\
\hline Ross River virus ${ }^{b}$ & 45 & 52 & 76 & 102 & 113 & 66 & 42 & 46 & 61 & 76 & 84 & 78 & 841 \\
\hline Other ${ }^{b}$ & 9 & 10 & 11 & 7 & 6 & 4 & 9 & 7 & 1 & 4 & 5 & 11 & 84 \\
\hline Blood lead level $\geq 15 \mathrm{ug} / \mathrm{dL}^{\mathrm{b}}$ & 7 & 7 & 26 & 9 & 24 & 15 & 38 & 47 & 36 & 23 & 20 & 11 & 263 \\
\hline Botulism & 0 & 0 & 0 & 0 & 0 & 0 & 0 & 0 & 0 & 0 & 0 & 0 & 0 \\
\hline Brucellosis $^{b}$ & 1 & 1 & 1 & 0 & 0 & 0 & 0 & 0 & 0 & 0 & 0 & 0 & 3 \\
\hline Chancroid $^{\mathrm{b}}$ & 0 & 0 & 0 & 0 & 0 & 0 & 0 & 0 & 0 & 0 & 0 & 0 & 0 \\
\hline Chlamydia trachomatis infection & 1039 & 1196 & 1203 & 913 & 1080 & 958 & 1011 & 970 & 927 & 1138 & 1157 & 855 & 12447 \\
\hline Congenital chlamydia ${ }^{b}$ & 2 & 2 & 3 & 3 & 3 & 2 & 4 & 3 & 3 & 0 & 3 & 3 & 31 \\
\hline Chlamydia - other ${ }^{b}$ & 1037 & 1194 & 1200 & 910 & 1077 & 956 & 1007 & 967 & 924 & 1138 & 1154 & 852 & 12416 \\
\hline Cholera $^{\mathrm{b}}$ & 0 & 0 & 1 & 0 & 0 & 1 & 0 & 0 & 0 & 0 & 0 & 0 & 2 \\
\hline Creutzfeldt-Jakob disease $^{b}$ & 1 & 0 & 0 & 3 & 0 & 2 & 1 & 0 & 0 & 0 & 0 & 0 & 7 \\
\hline Cryptosporidiosis $^{\mathrm{b}}$ & 37 & 42 & 25 & 32 & 33 & 16 & 18 & 15 & 22 & 35 & 157 & 112 & 544 \\
\hline Foodborne illness (NOS)e & 77 & 67 & 395 & 21 & 38 & 21 & 30 & 15 & 34 & 38 & 27 & 0 & 763 \\
\hline Gastroenteritis (institutional) & 154 & 221 & 423 & 438 & 562 & 873 & 1794 & 1471 & 1543 & 1679 & 935 & 395 & 10488 \\
\hline Giardiasis $^{b}$ & 161 & 191 & 245 & 151 & 185 & 159 & 147 & 149 & 122 & 122 & 190 & 118 & 1940 \\
\hline Gonorrhoeab & 144 & 119 & 133 & 118 & 132 & 116 & 87 & 91 & 96 & 125 & 98 & 125 & 1384 \\
\hline Haemolytic uraemic syndrome & 2 & 1 & 1 & 1 & 0 & 0 & 1 & 0 & 1 & 1 & 2 & 3 & 13 \\
\hline Haemophilus influenzae serotype b & 0 & 0 & 0 & 0 & 0 & 1 & 1 & 2 & 0 & 1 & 2 & 0 & 7 \\
\hline Hib epiglottitisb & 0 & 0 & 0 & 0 & 0 & 0 & 0 & 0 & 0 & 0 & 1 & 0 & 1 \\
\hline Hib meningitis ${ }^{b}$ & 0 & 0 & 0 & 0 & 0 & 1 & 0 & 0 & 0 & 0 & 1 & 0 & 2 \\
\hline Hib septicaemiab & 0 & 0 & 0 & 0 & 0 & 0 & 1 & 1 & 0 & 0 & 0 & 0 & 2 \\
\hline Hib infection NOS & 0 & 0 & 0 & 0 & 0 & 0 & 0 & 1 & 0 & 1 & 0 & 0 & 2 \\
\hline Hepatitis $A^{b}$ & 9 & 11 & 3 & 3 & 4 & 5 & 8 & 6 & 2 & 3 & 5 & 6 & 65 \\
\hline Hepatitis B & 242 & 204 & 268 & 224 & 224 & 219 & 232 & 215 & 205 & 210 & 240 & 173 & 2656 \\
\hline Hepatitis B - acute viral ${ }^{b}$ & 9 & 4 & 4 & 3 & 8 & 7 & 2 & 1 & 5 & 1 & 11 & 1 & 56 \\
\hline Hepatitis B - other ${ }^{\mathrm{b}}$ & 233 & 200 & 264 & 221 & 216 & 212 & 230 & 214 & 200 & 209 & 229 & 172 & 2600 \\
\hline Hepatitis C & 372 & 344 & 422 & 323 & 399 & 330 & 331 & 384 & 357 & 378 & 346 & 273 & 4259 \\
\hline Hepatitis C - acute viral ${ }^{b}$ & 4 & 9 & 3 & 4 & 7 & 3 & 8 & 7 & 4 & 2 & 2 & 0 & 53 \\
\hline Hepatitis C - other ${ }^{b}$ & 368 & 335 & 419 & 319 & 392 & 327 & 323 & 377 & 353 & 376 & 344 & 273 & 4206 \\
\hline Hepatitis $D^{b}$ & 2 & 0 & 1 & 2 & 2 & 0 & 2 & 0 & 1 & 1 & 0 & 0 & 11 \\
\hline Hepatitis $E^{b}$ & 0 & 0 & 4 & 0 & 0 & 0 & 1 & 1 & 0 & 1 & 0 & 1 & 8 \\
\hline HIV infection ${ }^{b}$ & 34 & 45 & 32 & 34 & 47 & 23 & 31 & 35 & 27 & 31 & 35 & 30 & 404 \\
\hline Influenza & 37 & 33 & 37 & 51 & 26 & 90 & 583 & 754 & 179 & 66 & 39 & 23 & 1918 \\
\hline Influenza-Type $\mathrm{A}^{\mathrm{b}}$ & 29 & 18 & 30 & 30 & 15 & 68 & 526 & 601 & 102 & 35 & 24 & 9 & 1487 \\
\hline Influenza-Type $\mathrm{B}^{\mathrm{b}}$ & 3 & 12 & 5 & 8 & 5 & 10 & 27 & 38 & 29 & 22 & 13 & 8 & 180 \\
\hline Influenza-Type A \& B b & 3 & 0 & 2 & 10 & 3 & 6 & 4 & 6 & 5 & 3 & 1 & 0 & 43 \\
\hline Influenza-Type NOS & 2 & 3 & 0 & 3 & 3 & 6 & 26 & 109 & 43 & 6 & 1 & 6 & 208 \\
\hline Legionellosis & 14 & 12 & 8 & 13 & 7 & 10 & 7 & 2 & 4 & 4 & 12 & 12 & 105 \\
\hline Legionella longbeachae & 3 & 5 & 3 & 6 & 1 & 2 & 3 & 0 & 1 & 1 & 2 & 2 & 29 \\
\hline L.pneumophilab & 11 & 7 & 5 & 7 & 6 & 8 & 4 & 1 & 3 & 3 & 8 & 10 & 73 \\
\hline Legionnaire disease other & 0 & 0 & 0 & 0 & 0 & 0 & 0 & 1 & 0 & 0 & 2 & 0 & 3 \\
\hline Leprosy & 1 & 1 & 1 & 0 & 0 & 0 & 0 & 0 & 0 & 0 & 0 & 1 & 4 \\
\hline Leptospirosis $^{\mathrm{b}}$ & 1 & 1 & 3 & 0 & 0 & 0 & 0 & 1 & 1 & 0 & 1 & 0 & 8 \\
\hline Listeriosis $^{\mathrm{b}}$ & 3 & 2 & 1 & 3 & 1 & 1 & 2 & 2 & 1 & 0 & 4 & 2 & 22 \\
\hline Lymphogranuloma venereum (LGV) ${ }^{\mathrm{b}}$ & 0 & 0 & 0 & 0 & 0 & 0 & 0 & 0 & 0 & 0 & 0 & 0 & 0 \\
\hline Malaria ${ }^{\mathrm{b}}$ & 9 & 9 & 10 & 5 & 8 & 6 & 5 & 12 & 10 & 12 & 7 & 5 & 98 \\
\hline Measles & 0 & 1 & 1 & 0 & 1 & 0 & 1 & 0 & 0 & 0 & 0 & 0 & 4 \\
\hline Measles laboratory confirmed & 0 & 1 & 1 & 0 & 1 & 0 & 1 & 0 & 0 & 0 & 0 & 0 & 4 \\
\hline Measles - other & 0 & 0 & 0 & 0 & 0 & 0 & 0 & 0 & 0 & 0 & 0 & 0 & 0 \\
\hline Meningococcal disease & 7 & 0 & 10 & 5 & 7 & 9 & 13 & 21 & 9 & 12 & 8 & 11 & 112 \\
\hline Meningococcal - serogroup $\mathrm{B}^{\mathrm{b}}$ & 6 & 0 & 4 & 2 & 2 & 5 & 8 & 17 & 8 & 7 & 7 & 10 & 76 \\
\hline Meningococcal - serogroup $C^{b}$ & 1 & 0 & 3 & 2 & 2 & 0 & 0 & 0 & 1 & 0 & 1 & 0 & 10 \\
\hline Meningococcal - serogroup W135 & 0 & 0 & 1 & 0 & 0 & 1 & 0 & 0 & 0 & 0 & 0 & 0 & 2 \\
\hline Meningococcal - serogroup $\mathrm{Yb}^{\mathrm{b}}$ & 0 & 0 & 0 & 0 & 0 & 2 & 1 & 0 & 0 & 1 & 0 & 1 & 5 \\
\hline Meningococcal - other & 0 & 0 & 2 & 1 & 3 & 1 & 4 & 4 & 0 & 4 & 0 & 0 & 19 \\
\hline Mumps ${ }^{\mathrm{b}}$ & 15 & 4 & 5 & 11 & 29 & 17 & 15 & 27 & 38 & 56 & 59 & 47 & 323 \\
\hline Pertussis & 122 & 136 & 100 & 112 & 167 & 174 & 211 & 180 & 177 & 268 & 255 & 191 & 2093 \\
\hline Pneumococcal disease (invasive) ${ }^{b}$ & 25 & 18 & 30 & 36 & 35 & 61 & 85 & 78 & 48 & 43 & 38 & 25 & 522 \\
\hline Psittacosis ${ }^{b}$ & 5 & 3 & 3 & 5 & 5 & 1 & 0 & 2 & 1 & 5 & 3 & 1 & 34 \\
\hline Q fever ${ }^{b}$ & 23 & 18 & 14 & 14 & 20 & 15 & 15 & 15 & 16 & 21 & 24 & 20 & 215 \\
\hline Rubella & 0 & 2 & 1 & 0 & 1 & 4 & 0 & 0 & 0 & 0 & 1 & 0 & 9 \\
\hline Congenital rubellab & 0 & 0 & 0 & 0 & 0 & 1 & 0 & 0 & 0 & 0 & 0 & 0 & 1 \\
\hline Rubella - other ${ }^{b}$ & 0 & 2 & 1 & 0 & 1 & 3 & 0 & 0 & 0 & 0 & 1 & 0 & 8 \\
\hline Salmonella infection ${ }^{b, d}$ & 233 & 314 & 510 & 317 & 186 & 126 & 108 & 114 & 115 & 155 & 202 & 184 & 2564 \\
\hline Shigellosis ${ }^{b}$ & 4 & 4 & 9 & 7 & 8 & 4 & 6 & 12 & 3 & 4 & 4 & 6 & 71 \\
\hline Syphilis & 93 & 93 & 107 & 78 & 105 & 97 & 78 & 118 & 83 & 85 & 98 & 80 & 1115 \\
\hline Congenital syphilis & 0 & 1 & 1 & 0 & 0 & 0 & 0 & 0 & 0 & 0 & 1 & 1 & 4 \\
\hline Infectious syphilis $b, c$ & 40 & 38 & 29 & 33 & 41 & 43 & 35 & 41 & 27 & 30 & 49 & 28 & 434 \\
\hline Syphilis - other ${ }^{b}$ & 53 & 54 & 77 & 45 & 64 & 54 & 43 & 77 & 56 & 55 & 48 & 51 & 677 \\
\hline Tetanus & 0 & 0 & 0 & 0 & 0 & 0 & 1 & 0 & 0 & 0 & 0 & 1 & 2 \\
\hline Tuberculosis ${ }^{b}$ & 54 & 45 & 49 & 38 & 27 & 46 & 31 & 36 & 37 & 41 & 28 & 20 & 452 \\
\hline Typhoid ${ }^{b}$ & 2 & 6 & 3 & 6 & 2 & 1 & 3 & 2 & 0 & 0 & 1 & 0 & 26 \\
\hline $\begin{array}{l}\text { Verotoxin - producing } \\
\text { Escherichia coli infections }{ }^{b}\end{array}$ & 1 & 1 & 1 & 1 & 1 & 0 & 0 & 0 & 0 & 5 & 8 & 5 & 23 \\
\hline
\end{tabular}

aYear of onset: the earlier of patient reported onset date, specimen date or date of notification. bLaboratory-confirmed cases only. Includes Syphilis primary, Syphilis secondary, Syphilis < 1 year duration and Syphilis newly acquired. dIncludes all paratyphoid cases. eFoodborne illness cases are only those notified as part of an outbreak. NOS: not otherwise specified. No case of the following diseases have been notified since 1991: Plague ${ }^{\mathrm{b}}$, Diphtheria ${ }^{\mathrm{b}}$, Granuloma inguinale ${ }^{\mathrm{b}}$, Lyssavirusb, Poliomyelitis ${ }^{\mathrm{b}}$, Rabies, Smallpox, Typhus ${ }^{b}$, Viral haemorrhagic fever, Yellow fever. Due to data delay AIDS notifications will be reported in a later edition. 
Table 4. Disease notifications by Area Health Service of residence (2005 AHS boundaries), crude rates per 100000 population, NSW, 2007

\begin{tabular}{|c|c|c|c|c|c|c|c|c|c|}
\hline \multirow[t]{2}{*}{ Condition } & \multicolumn{2}{|c|}{ Greater Southern $\mathrm{f}^{\mathrm{s}}$} & \multicolumn{3}{|c|}{ Greater Western ${ }^{f}$} & \multicolumn{2}{|c|}{ Hunter New Englandf } & \multicolumn{2}{|c|}{ North Coast ${ }^{f}$} \\
\hline & Albury & Goulburn & Broken Hill & Dubbo & Bathurst & Newcastle & Tamworth & Port Macquarie & Lismore \\
\hline Adverse event after immunisation & 7.12 & 8.14 & 2.22 & 4.83 & 5.79 & 2.57 & 1.68 & 1.39 & 2.46 \\
\hline Anthrax & 0 & 0 & 0 & 0 & 0 & 0 & 0 & 0 & 0 \\
\hline Arboviral infection & 22.11 & 64.13 & 66.71 & 84.07 & 15.62 & 57.46 & 38.05 & 76.72 & 74.85 \\
\hline Barmah Forest virus ${ }^{b}$ & 2.62 & 50.25 & 4.45 & 9.66 & 2.31 & 20.24 & 9.51 & 31.24 & 39 \\
\hline Ross River virus ${ }^{\mathrm{b}}$ & 19.12 & 12.92 & 62.26 & 73.44 & 12.73 & 36.88 & 27.42 & 44.09 & 31.98 \\
\hline Other ${ }^{b}$ & 0.37 & 0.96 & 0 & 0.97 & 0.58 & 0.34 & 1.12 & 1.39 & 3.87 \\
\hline Blood lead level $\geq 15 \mathrm{ug} / \mathrm{dL}^{\mathrm{b}}$ & 3 & 1.91 & 11.12 & 71.5 & 4.63 & 3.77 & 0.56 & 0.69 & 1.05 \\
\hline Botulism & 0 & 0 & 0 & 0 & 0 & 0 & 0 & 0 & 0 \\
\hline Brucellosis $^{b}$ & 0 & 0 & 0 & 0 & 0.58 & 0 & 0 & 0 & 0 \\
\hline Chancroid ${ }^{b}$ & 0 & 0 & 0 & 0 & 0 & 0 & 0 & 0 & 0 \\
\hline Chlamydia trachomatis infection & 176.6 & 126.3 & 231.3 & 143 & 206.6 & 229 & 232.2 & 123.6 & 217.2 \\
\hline Congenital chlamydia ${ }^{b}$ & 0.37 & 0.96 & 2.22 & 0 & 0.58 & 0.34 & 0 & 0.35 & 0.7 \\
\hline Chlamydia - other ${ }^{\mathrm{b}}$ & 176.2 & 125.4 & 229 & 143 & 206 & 228.7 & 232.2 & 123.2 & 216.5 \\
\hline Cholera ${ }^{\mathrm{b}}$ & 0 & 0 & 0 & 0 & 0 & 0 & 0 & 0 & 0 \\
\hline Creutzfeldt-Jakob disease $^{b}$ & 0 & 0 & 0 & 0 & 0 & 0.17 & 0 & 0 & 0 \\
\hline Cryptosporidiosis $^{\mathrm{b}}$ & 19.49 & 6.7 & 4.45 & 16.43 & 19.1 & 7.21 & 35.26 & 9.37 & 17.22 \\
\hline Giardiasis $^{\mathrm{b}}$ & 17.62 & 15.31 & 8.89 & 42.52 & 16.78 & 28.65 & 33.02 & 19.09 & 5.97 \\
\hline Gonorrhoea $^{b}$ & 5.25 & 1.91 & 0 & 3.87 & 6.37 & 12.87 & 5.6 & 2.43 & 14.76 \\
\hline Haemolytic uraemic syndrome & 0 & 0 & 0 & 0 & 0.58 & 0.86 & 0.56 & 0 & 0 \\
\hline H.influenzae serotype b & 0.37 & 0 & 0 & 0 & 0 & 0.17 & 0 & 0.35 & 0 \\
\hline Hib epiglottitis ${ }^{b}$ & 0 & 0 & 0 & 0 & 0 & 0 & 0 & 0 & 0 \\
\hline Hib meningitis ${ }^{b}$ & 0.37 & 0 & 0 & 0 & 0 & 0 & 0 & 0.35 & 0 \\
\hline Hib septicaemiab & 0 & 0 & 0 & 0 & 0 & 0.17 & 0 & 0 & 0 \\
\hline Hib infection NOSb & 0 & 0 & 0 & 0 & 0 & 0 & 0 & 0 & 0 \\
\hline Hepatitis $A^{b}$ & 0 & 0 & 0 & 0.97 & 0.58 & 0.17 & 0 & 0.35 & 1.76 \\
\hline Hepatitis B & 13.49 & 11.01 & 22.24 & 9.67 & 1.16 & 8.4 & 10.63 & 5.9 & 12.65 \\
\hline Hepatitis B - acute viral ${ }^{\mathrm{b}}$ & 0.75 & 1.44 & 0 & 0.97 & 0 & 1.37 & 0 & 0 & 0.7 \\
\hline Hepatitis B - other ${ }^{\mathrm{b}}$ & 12.74 & 9.57 & 22.24 & 8.7 & 1.16 & 7.03 & 10.63 & 5.9 & 11.95 \\
\hline Hepatitis C & 38.98 & 55.04 & 77.82 & 68.6 & 61.34 & 55.07 & 50.93 & 48.25 & 76.25 \\
\hline Hepatitis C - acute viral ${ }^{b}$ & 0.37 & 1.44 & 6.67 & 4.83 & 0.58 & 0.69 & 1.68 & 0 & 0 \\
\hline Hepatitis C - other ${ }^{b}$ & 38.61 & 53.6 & 71.15 & 63.77 & 60.76 & 54.38 & 49.25 & 48.25 & 76.25 \\
\hline Hepatitis $D^{b}$ & 0 & 0 & 0 & 0 & 0 & 0 & 0 & 0 & 0 \\
\hline Hepatitis $E^{b}$ & 0 & 0 & 0 & 0 & 0 & 0 & 0 & 0 & 0 \\
\hline HIV infection ${ }^{b}$ & 0.75 & 1.44 & 0 & 1.93 & 1.16 & 3.09 & 0.56 & 1.39 & 1.41 \\
\hline Influenza & 14.98 & 35.9 & 22.24 & 19.33 & 37.04 & 37.22 & 45.33 & 15.27 & 58.32 \\
\hline Influenza-Type $\mathrm{A}^{\mathrm{b}}$ & 13.87 & 33.5 & 22.24 & 16.43 & 34.72 & 32.42 & 40.85 & 14.23 & 21.43 \\
\hline Influenza-Type $\mathrm{B}^{\mathrm{b}}$ & 0.37 & 1.44 & 0 & 2.9 & 1.74 & 4.8 & 3.36 & 0 & 1.05 \\
\hline Influenza-Type A \& B b & 0.37 & 0.96 & 0 & 0 & 0 & 0 & 0.56 & 0 & 1.05 \\
\hline Influenza-Type NOS & 0.37 & 0 & 0 & 0 & 0.58 & 0 & 0.56 & 1.04 & 34.79 \\
\hline Legionellosis & 1.12 & 2.39 & 0 & 0 & 0 & 0.85 & 2.24 & 1.38 & 1.05 \\
\hline L. longbeachae b & 0.37 & 0.48 & 0 & 0 & 0 & 0.34 & 1.12 & 0.69 & 0 \\
\hline L. pneumophilab & 0 & 1.91 & 0 & 0 & 0 & 0.51 & 0.56 & 0.69 & 1.05 \\
\hline Legionnaire disease other & 0.75 & 0 & 0 & 0 & 0 & 0 & 0.56 & 0 & 0 \\
\hline Leprosy & 0 & 0 & 0 & 0 & 0 & 0 & 0 & 0 & 0 \\
\hline Leptospirosis ${ }^{b}$ & 0 & 0 & 0 & 0.97 & 0 & 0.17 & 0.56 & 0.69 & 1.05 \\
\hline Listeriosis ${ }^{\mathrm{b}}$ & 0 & 0 & 2.22 & 0 & 0 & 0.86 & 0 & 0 & 0 \\
\hline Lymphogranuloma venereum (LGV)b & 0 & 0 & 0 & 0 & 0 & 0 & 0 & 0 & 0 \\
\hline Malariab & 1.12 & 2.87 & 0 & 0 & 0.58 & 2.4 & 1.12 & 1.39 & 0.7 \\
\hline Measles & 0 & 0 & 0 & 0 & 0 & 0 & 0 & 0 & 0 \\
\hline Measles laboratory confirmed & 0 & 0 & 0 & 0 & 0 & 0 & 0 & 0 & 0 \\
\hline Measles - other & 0 & 0 & 0 & 0 & 0 & 0 & 0 & 0 & 0 \\
\hline Meningococcal disease & 1.49 & 2.87 & 0 & 2.9 & 1.16 & 1.54 & 1.68 & 0.69 & 2.1 \\
\hline Meningococcal - serogroup $\mathrm{B}^{\mathrm{b}}$ & 0.75 & 2.39 & 0 & 2.9 & 1.16 & 1.03 & 1.12 & 0.69 & 1.05 \\
\hline Meningococcal - serogroup $C^{b}$ & 0.37 & 0 & 0 & 0 & 0 & 0.17 & 0 & 0 & 0.7 \\
\hline Meningococcal - serogroup W135 & 0 & 0.48 & 0 & 0 & 0 & 0 & 0 & 0 & 0 \\
\hline Meningococcal - serogroup $\mathrm{Y}^{\mathrm{b}}$ & 0 & 0 & 0 & 0 & 0 & 0 & 0 & 0 & 0 \\
\hline Meningococcal - other & 0.37 & 0 & 0 & 0 & 0 & 0.34 & 0.56 & 0 & 0.35 \\
\hline Mumps $^{\mathrm{b}}$ & 0.75 & 0 & 0 & 0 & 1.16 & 0.86 & 0.56 & 0 & 0 \\
\hline Pertussis & 23.99 & 26.32 & 13.34 & 55.08 & 10.42 & 34.31 & 35.81 & 16.66 & 32.33 \\
\hline Pneumococcal disease (invasive) & 7.87 & 6.7 & 15.57 & 14.49 & 6.94 & 10.98 & 9.51 & 9.03 & 7.03 \\
\hline Psittacosis ${ }^{b}$ & 1.5 & 0.48 & 2.22 & 1.93 & 1.74 & 0.86 & 0 & 0.35 & 0.7 \\
\hline Q fever ${ }^{\mathrm{b}}$ & 1.5 & 6.22 & 6.67 & 44.45 & 4.63 & 3.6 & 31.9 & 5.55 & 9.49 \\
\hline Rubella & 0 & 0 & 0 & 2.9 & 0 & 0 & 0.56 & 0 & 0 \\
\hline Congenital rubellab & 0 & 0 & 0 & 0 & 0 & 0 & 0 & 0 & 0 \\
\hline Rubella - other ${ }^{\mathrm{b}}$ & 0 & 0 & 0 & 2.9 & 0 & 0 & 0.56 & 0 & 0 \\
\hline Salmonella infection ${ }^{\mathrm{b}, \mathrm{d}}$ & 31.86 & 26.8 & 15.57 & 27.06 & 27.2 & 32.59 & 43.65 & 27.08 & 76.6 \\
\hline Shigellosis ${ }^{b}$ & 0 & 1.44 & 0 & 0.97 & 0 & 0.51 & 0.56 & 1.04 & 2.81 \\
\hline Syphilis & 3.37 & 3.83 & 31.13 & 15.46 & 9.26 & 4.29 & 4.48 & 9.37 & 4.22 \\
\hline Congenital syphilis & 0 & 0 & 0 & 0 & 0.58 & 0 & 0 & 0 & 0 \\
\hline Infectious syphilis, & 0.37 & 0.96 & 2.22 & 0 & 1.74 & 2.06 & 1.12 & 0.69 & 1.76 \\
\hline Syphilis - other ${ }^{b}$ & 3 & 2.87 & 28.91 & 15.46 & 6.94 & 2.23 & 3.36 & 8.68 & 2.46 \\
\hline Tetanus & 0 & 0 & 0 & 0 & 0 & 0 & 0 & 0 & 0.35 \\
\hline Tuberculosis ${ }^{b}$ & 1.12 & 2.87 & 0 & 0 & 0.58 & 2.74 & 0.56 & 1.39 & 1.41 \\
\hline Typhoid $^{\mathrm{b}}$ & 0.37 & 0 & 0 & 0 & 0 & 0 & 0 & 0 & 0 \\
\hline Verotoxin-producing Escherichia coli infections ${ }^{b}$ & 1.12 & 0.48 & 0 & 0 & 0 & 1.54 & 2.24 & 0 & 0.35 \\
\hline
\end{tabular}

aYear of onset: the earlier of patient reported onset date, specimen date or date of notification. ' ${ }^{2}$ Laboratory-confirmed cases only. CIncludes Syphilis primary, Syphilis secondary, Syphilis $<1$ year duration and Syphilis newly acquired. IIncludes all paratyphoid cases. ${ }^{\mathrm{f}} \mathrm{AHS}$ further divided into the geographical region covered by their component Public Health Unit. 9Rate is based on a denominator of 8000 persons. hIncludes cases with unknown PHU. NOS: not otherwise specified. No case of the following diseases have been notified since 1991: Plague $^{\mathrm{b}}$, Diphtheriab, Granuloma inguinale ${ }^{\mathrm{b}}$, Lyssavirus ${ }^{\mathrm{b}}$, Poliomyelitis ${ }^{\mathrm{b}}$, Rabies, Smallpox, Typhus $\mathrm{s}^{\mathrm{b}}$, Viral haemorrhagic fever, Yellow fever. Due to data delay AIDS notifications will be reported in a later edition. 
Table 4. continued

\begin{tabular}{|c|c|c|c|c|c|c|c|c|c|}
\hline \multirow[t]{2}{*}{ Condition } & \multicolumn{2}{|c|}{ Northern Sydney Central Coast ${ }^{f}$} & \multicolumn{2}{|c|}{ South Eastern Sydney Illawara ${ }^{f}$} & \multicolumn{2}{|c|}{ Sydney South West ${ }^{f}$} & \multicolumn{2}{|c|}{ Sydney West ${ }^{f}$} & \multirow{2}{*}{$\begin{array}{l}\text { Justice } \\
\text { Health }\end{array}$} \\
\hline & Gosford & Hornsby & Wollongong & Randwick & Camperdown & Liverpool & Penrith & Parramatta & \\
\hline Adverse event after immunisation & 4.84 & 1.73 & 5.08 & 3.07 & 1.14 & 1.93 & 5.98 & 3.63 & 0 \\
\hline Anthrax & 0 & 0 & 0 & 0 & 0 & 0 & 0 & 0 & 0 \\
\hline Arboviral infection & 21.63 & 4.96 & 26.74 & 5.28 & 3.42 & 1.68 & 4.41 & 3.12 & 12.5 \\
\hline Barmah Forest virus ${ }^{b}$ & 5.17 & 0.74 & 18.45 & 0.49 & 0.57 & 0.24 & 0.63 & 0.78 & 12.5 \\
\hline Ross River virus ${ }^{b}$ & 14.85 & 2.73 & 7.49 & 2.09 & 1.52 & 0.84 & 3.15 & 1.95 & 0 \\
\hline Otherb & 1.61 & 1.49 & 0.8 & 2.7 & 1.33 & 0.6 & 0.63 & 0.39 & 0 \\
\hline Blood lead level $\geq 15 \mathrm{ug} / \mathrm{dL}^{\mathrm{b}}$ & 0.97 & 1.24 & 5.61 & 1.59 & 2.66 & 2.77 & 2.52 & 3.89 & 0 \\
\hline Botulism & 0 & 0 & 0 & 0 & 0 & 0 & 0 & 0 & 0 \\
\hline Brucellosis $^{b}$ & 0 & 0 & 0 & 0.12 & 0 & 0.12 & 0 & 0 & 0 \\
\hline Chancroid $^{b}$ & 0 & 0 & 0 & 0 & 0 & 0 & 0 & 0 & 0 \\
\hline Chlamydia trachomatis infection & 186.9 & 133.3 & 154.6 & 280.1 & 253.8 & 99.9 & 128.8 & 135.1 & 1188 \\
\hline Congenital chlamydiab & 0.32 & 0.37 & 0 & 0.12 & 0.19 & 0.36 & 0.63 & 1.17 & 0 \\
\hline Chlamydia - other ${ }^{b}$ & 186.6 & 132.9 & 154.6 & 279.9 & 253.6 & 99.54 & 128.2 & 134 & 1188 \\
\hline Cholerab $^{b}$ & 0 & 0.12 & 0 & 0 & 0.19 & 0 & 0 & 0 & 0 \\
\hline Creutzfeldt-Jakob disease ${ }^{b}$ & 0.32 & 0 & 0.53 & 0.12 & 0 & 0.12 & 0.31 & 0 & 0 \\
\hline Cryptosporidiosis $^{\mathrm{b}}$ & 7.1 & 5.33 & 4.01 & 4.66 & 4.18 & 5.79 & 6.61 & 4.41 & 0 \\
\hline Giardiasis $^{\mathrm{b}}$ & 26.15 & 38.77 & 22.19 & 40.6 & 38.21 & 14.36 & 26.76 & 34.62 & 25 \\
\hline Gonorrhoeab $^{b}$ & 9.36 & 15.48 & 8.56 & 57.53 & 56.46 & 11.58 & 11.97 & 12.45 & 100 \\
\hline Haemolytic uraemic syndrome & 0 & 0 & 0.27 & 0.12 & 0.19 & 0.12 & 0 & 0.13 & 0 \\
\hline H.influenzae serotype b & 0 & 0.12 & 0 & 0.12 & 0 & 0.12 & 0 & 0.13 & 0 \\
\hline Hib epiglottitis ${ }^{b}$ & 0 & 0.12 & 0 & 0 & 0 & 0 & 0 & 0 & 0 \\
\hline Hib meningitis ${ }^{b}$ & 0 & 0 & 0 & 0 & 0 & 0 & 0 & 0 & 0 \\
\hline Hib septicaemiab & 0 & 0 & 0 & 0 & 0 & 0 & 0 & 0.13 & 0 \\
\hline Hib infection NOSb & 0 & 0 & 0 & 0.12 & 0 & 0.12 & 0 & 0 & 0 \\
\hline Hepatitis $A^{b}$ & 0 & 1.61 & 0.8 & 1.23 & 1.14 & 1.45 & 0.94 & 1.17 & 0 \\
\hline Hepatitis B & 10.66 & 35.42 & 12.3 & 47.35 & 81.17 & 72.39 & 12.28 & 72.23 & 550 \\
\hline Hepatitis B - acute viral ${ }^{b}$ & 0.65 & 0.74 & 0 & 1.72 & 0.57 & 1.45 & 0.31 & 0.13 & 12.5 \\
\hline Hepatitis B - other ${ }^{b}$ & 10.01 & 34.68 & 12.3 & 45.63 & 80.6 & 70.94 & 11.97 & 72.1 & 537.5 \\
\hline Hepatitis C & 60.05 & 25.15 & 49.47 & 48.46 & 75.85 & 59.72 & 50.06 & 49.15 & 7675 \\
\hline Hepatitis C - acute viralb & 0 & 0 & 0 & 0.25 & 3.99 & 0.6 & 0 & 0.13 & 50 \\
\hline Hepatitis C - other ${ }^{b}$ & 60.05 & 25.15 & 49.47 & 48.21 & 71.86 & 59.12 & 50.06 & 49.02 & 7625 \\
\hline Hepatitis $D^{b}$ & 0.32 & 0 & 0.53 & 0.37 & 0 & 0 & 0.31 & 0.52 & 0 \\
\hline Hepatitis $E^{b}$ & 0.32 & 0.12 & 0 & 0.37 & 0.57 & 0 & 0 & 0 & 0 \\
\hline HIV infection ${ }^{b}$ & 2.58 & 3.84 & 2.41 & 15.7 & 18.44 & 2.9 & 0.94 & 3.89 & 0 \\
\hline Influenza & 17.43 & 17.59 & 16.31 & 22.69 & 8.55 & 14.12 & 39.04 & 60.04 & 37.5 \\
\hline Influenza-Type $A^{b}$ & 12.27 & 10.16 & 12.3 & 14.35 & 5.51 & 9.29 & 31.49 & 55.89 & 37.5 \\
\hline Influenza-Type $\mathrm{B}^{\mathrm{b}}$ & 0.32 & 0.74 & 2.41 & 6.38 & 2.85 & 1.21 & 4.41 & 3.37 & 0 \\
\hline Influenza-Type $A$ \& $B^{b}$ & 0 & 0.25 & 1.07 & 1.96 & 0 & 0 & 2.83 & 0.65 & 0 \\
\hline Influenza-Type NOSb & 4.84 & 6.44 & 0.53 & 0 & 0.19 & 3.62 & 0.31 & 0.13 & 0 \\
\hline Legionellosis & 1.3 & 0.99 & 1.6 & 1.6 & 1.52 & 1.57 & 3.15 & 2.47 & 0 \\
\hline L.longbeachae ${ }^{b}$ & 0.65 & 0.25 & 1.07 & 0.25 & 0 & 0.48 & 0.63 & 0.65 & 0 \\
\hline L.pneumophilab & 0.65 & 0.74 & 0.53 & 1.35 & 1.52 & 1.09 & 2.52 & 1.82 & 0 \\
\hline Legionnaire disease other & 0 & 0 & 0 & 0 & 0 & 0 & 0 & 0 & 0 \\
\hline Leprosy & 0 & 0 & 0 & 0 & 0 & 0 & 0 & 0.52 & 0 \\
\hline Leptospirosis ${ }^{b}$ & 0 & 0 & 0 & 0 & 0 & 0 & 0 & 0 & 0 \\
\hline Listeriosis $^{\mathrm{b}}$ & 0 & 0.37 & 0.53 & 0.61 & 0.19 & 0.24 & 0.31 & 0.26 & 0 \\
\hline Lymphogranuloma venereum (LGV) & 0 & 0 & 0 & 0 & 0 & 0 & 0 & 0 & 0 \\
\hline Malariab & 0.65 & 1.24 & 1.6 & 0.98 & 1.71 & 0.6 & 1.89 & 2.07 & 0 \\
\hline Measles & 0 & 0.12 & 0 & 0.12 & 0 & 0.12 & 0 & 0 & 0 \\
\hline Measles laboratory confirmed & 0 & 0.12 & 0 & 0.12 & 0 & 0.12 & 0 & 0 & 0 \\
\hline Measles - other & 0 & 0 & 0 & 0 & 0 & 0 & 0 & 0 & 0 \\
\hline Meningococcal disease & 1.94 & 1.6 & 1.07 & 2.08 & 0.95 & 1.45 & 1.57 & 1.82 & 0 \\
\hline Meningococcal - serogroup $\mathrm{B}^{\mathrm{b}}$ & 0.97 & 1.36 & 0.8 & 1.1 & 0.76 & 1.09 & 1.26 & 1.04 & 0 \\
\hline Meningococcal - serogroup $\mathrm{C}^{\mathrm{b}}$ & 0.32 & 0 & 0.27 & 0.37 & 0.19 & 0 & 0 & 0 & 0 \\
\hline Meningococcal - serogroup W135 & 0 & 0 & 0 & 0 & 0 & 0.12 & 0 & 0 & 0 \\
\hline Meningococcal - serogroup $\mathrm{Y}^{\mathrm{b}}$ & 0.65 & 0.12 & 0 & 0.12 & 0 & 0 & 0 & 0.13 & 0 \\
\hline Meningococcal - other & 0 & 0.12 & 0 & 0.49 & 0 & 0.24 & 0.31 & 0.65 & 0 \\
\hline Mumps $^{\mathrm{b}}$ & 0.32 & 6.32 & 3.21 & 16.68 & 5.89 & 3.26 & 3.15 & 5.19 & 0 \\
\hline Pertussis & 24.54 & 34.81 & 20.86 & 39.13 & 33.65 & 18.46 & 27.39 & 41.11 & 0 \\
\hline Pneumococcal disease (invasive) ${ }^{b}$ & 7.1 & 6.94 & 9.63 & 6.13 & 7.79 & 5.31 & 7.87 & 6.22 & 12.5 \\
\hline Psittacosis ${ }^{b}$ & 0 & 0 & 0.8 & 0.25 & 0 & 0.24 & 1.57 & 0.39 & 0 \\
\hline Q fever ${ }^{\mathrm{b}}$ & 0.97 & 0.12 & 3.21 & 0.12 & 0 & 0 & 0.31 & 0.26 & 0 \\
\hline Rubella & 0 & 0.12 & 0 & 0 & 0.19 & 0 & 0 & 0.39 & 0 \\
\hline Congenital rubellab & 0 & 0 & 0 & 0 & 0 & 0 & 0 & 0.13 & 0 \\
\hline Rubella - other ${ }^{\mathrm{b}}$ & 0 & 0.12 & 0 & 0 & 0.19 & 0 & 0 & 0.26 & 0 \\
\hline Salmonella infection ${ }^{b, d}$ & 45.84 & 41.99 & 22.99 & 36.19 & 45.43 & 30.28 & 32.12 & 39.68 & 12.5 \\
\hline Shigellosis ${ }^{\mathrm{b}}$ & 0 & 1.61 & 0.27 & 1.84 & 2.28 & 0.72 & 0 & 0.52 & 0 \\
\hline Syphilis & 6.78 & 6.69 & 8.29 & 40.85 & 47.72 & 16.65 & 8.5 & 12.32 & 237.5 \\
\hline Congenital syphilis & 0 & 0.12 & 0 & 0 & 0 & 0 & 0 & 0.26 & 0 \\
\hline Infectious syphilis ${ }^{\mathrm{b}, \mathrm{c}}$ & 0.32 & 2.11 & 1.87 & 29.2 & 17.49 & 1.33 & 1.26 & 3.63 & 25 \\
\hline Syphilis - other ${ }^{b}$ & 6.46 & 4.46 & 6.42 & 11.65 & 30.23 & 15.32 & 7.24 & 8.43 & 212.5 \\
\hline Tetanus & 0 & 0 & 0.27 & 0 & 0 & 0 & 0 & 0 & 0 \\
\hline Tuberculosis ${ }^{b}$ & 1.29 & 5.33 & 2.67 & 7.73 & 12.93 & 9.77 & 4.09 & 16.6 & 0 \\
\hline Typhoid $^{\mathrm{b}}$ & 0 & 0.25 & 0 & 0.25 & 0.57 & 0.48 & 0 & 1.69 & 0 \\
\hline $\begin{array}{l}\text { Verotoxin-producing Escherichia } \\
\text { coli infections }{ }^{b}\end{array}$ & 0 & 0.12 & 0 & 0 & 0 & 0.12 & 0.63 & 0.13 & 0 \\
\hline
\end{tabular}

aYear of onset: the earlier of patient reported onset date, specimen date or date of notification. 'baboratory-confirmed cases only. CIncludes Syphilis primary, Syphilis secondary, Syphilis $<1$ year duration and Syphilis newly acquired. Includes all paratyphoid cases. ${ }^{\mathrm{f}} \mathrm{AHS}$ further divided into the geographical region covered by their component Public Health Unit.

gRate is based on a denominator of 8000 persons. hIncludes cases with unknown PHU. NOS: not otherwise specified. No case of the following diseases have been notified since 1991: Plague $^{\mathrm{b}}$, Diphtheria ${ }^{\mathrm{b}}$, Granuloma inguinale ${ }^{\mathrm{b}}$, Lyssavirus ${ }^{\mathrm{b}}$, Poliomyelitis ${ }^{\mathrm{b}}$, Rabies, Smallpox, Typhus ${ }^{\mathrm{b}}$, Viral haemorrhagic fever, Yellow fever.

Due to data delay AIDS notifications will be reported in a later edition. 
Table 5. Disease notifications by Area Health Service of residence (2005 AHS boundaries)

\begin{tabular}{|c|c|c|c|c|c|c|c|c|c|}
\hline \multirow[t]{2}{*}{ Condition } & \multicolumn{2}{|c|}{ Greater Southern ${ }^{f}$} & \multicolumn{3}{|c|}{ Greater Western ${ }^{f}$} & \multicolumn{2}{|c|}{ Hunter New England $d^{f}$} & \multicolumn{2}{|c|}{ North Coast ${ }^{f}$} \\
\hline & Albury & Goulburn & Broken Hill & Dubbo & Bathurst & Newcastle & Tamworth & Port Macquarie & Lismore \\
\hline Adverse event after immunisation & 19 & 17 & 1 & 5 & 10 & 15 & 3 & 4 & 7 \\
\hline Anthrax & 0 & 0 & 0 & 0 & 0 & 0 & 0 & 0 & 0 \\
\hline Arboviral infection & 59 & 134 & 30 & 87 & 27 & 335 & 68 & 221 & 213 \\
\hline Barmah Forest virusb & 7 & 105 & 2 & 10 & 4 & 118 & 17 & 90 & 111 \\
\hline Ross River virus ${ }^{b}$ & 51 & 27 & 28 & 76 & 22 & 215 & 49 & 127 & 91 \\
\hline Other ${ }^{b}$ & 1 & 2 & 0 & 1 & 1 & 2 & 2 & 4 & 11 \\
\hline Blood lead level $\geq 15 \mathrm{ug} / \mathrm{dL}^{\mathrm{b}}$ & 8 & 4 & 5 & 74 & 8 & 22 & 1 & 2 & 3 \\
\hline Botulism & 0 & 0 & 0 & 0 & 0 & 0 & 0 & 0 & 0 \\
\hline Brucellosis $^{b}$ & 0 & 0 & 0 & 0 & 1 & 0 & 0 & 0 & 0 \\
\hline Chancroid $^{b}$ & 0 & 0 & 0 & 0 & 0 & 0 & 0 & 0 & 0 \\
\hline Chlamydia trachomatis infection & 471 & 264 & 104 & 148 & 357 & 1335 & 415 & 356 & 618 \\
\hline Congenital chlamydia ${ }^{b}$ & 1 & 2 & 1 & 0 & 1 & 2 & 0 & 1 & 2 \\
\hline Chlamydia - other ${ }^{b}$ & 470 & 262 & 103 & 148 & 356 & 1333 & 415 & 355 & 616 \\
\hline Cholera $^{\mathrm{b}}$ & 0 & 0 & 0 & 0 & 0 & 0 & 0 & 0 & 0 \\
\hline Creutzfeldt-Jakob disease $^{\mathrm{b}}$ & 0 & 0 & 0 & 0 & 0 & 1 & 0 & 0 & 0 \\
\hline Cryptosporidiosis $^{\mathrm{b}}$ & 52 & 14 & 2 & 17 & 33 & 42 & 63 & 27 & 49 \\
\hline Gastroenteritis (institutional) & 120 & 583 & 247 & 38 & 60 & 1929 & 167 & 65 & 583 \\
\hline Giardiasis $^{b}$ & 47 & 32 & 4 & 44 & 29 & 167 & 59 & 55 & 17 \\
\hline Gonorrhoeab & 14 & 4 & 0 & 4 & 11 & 75 & 10 & 7 & 42 \\
\hline Haemolytic uraemic syndrome & 0 & 0 & 0 & 0 & 1 & 5 & 1 & 0 & 0 \\
\hline Haemophilus influenzae serotype $b$ & 1 & 0 & 0 & 0 & 0 & 1 & 0 & 1 & 0 \\
\hline Hib epiglottitis ${ }^{b}$ & 0 & 0 & 0 & 0 & 0 & 0 & 0 & 0 & 0 \\
\hline Hib meningitis ${ }^{b}$ & 1 & 0 & 0 & 0 & 0 & 0 & 0 & 1 & 0 \\
\hline Hib septicaemiab & 0 & 0 & 0 & 0 & 0 & 1 & 0 & 0 & 0 \\
\hline Hib infection NOSb & 0 & 0 & 0 & 0 & 0 & 0 & 0 & 0 & 0 \\
\hline Hepatitis $A^{b}$ & 0 & 0 & 0 & 1 & 1 & 1 & 0 & 1 & 5 \\
\hline Hepatitis B & 36 & 23 & 10 & 10 & 2 & 49 & 19 & 17 & 36 \\
\hline Hepatitis B - acute viral ${ }^{\mathrm{b}}$ & 2 & 3 & 0 & 1 & 0 & 8 & 0 & 0 & 2 \\
\hline Hepatitis B - other ${ }^{\mathrm{b}}$ & 34 & 20 & 10 & 9 & 2 & 41 & 19 & 17 & 34 \\
\hline Hepatitis C & 104 & 115 & 35 & 71 & 106 & 321 & 91 & 139 & 217 \\
\hline Hepatitis C - acute viral ${ }^{\mathrm{b}}$ & 1 & 3 & 3 & 5 & 1 & 4 & 3 & 0 & 0 \\
\hline Hepatitis C - other ${ }^{b}$ & 103 & 112 & 32 & 66 & 105 & 317 & 88 & 139 & 217 \\
\hline Hepatitis $D^{b}$ & 0 & 0 & 0 & 0 & 0 & 0 & 0 & 0 & 0 \\
\hline Hepatitis $E^{b}$ & 0 & 0 & 0 & 0 & 0 & 0 & 0 & 0 & 0 \\
\hline HIV infection ${ }^{b}$ & 2 & 3 & 0 & 2 & 2 & 18 & 1 & 4 & 4 \\
\hline Influenza & 40 & 75 & 10 & 20 & 64 & 217 & 81 & 44 & 166 \\
\hline Influenza-Type $A^{b}$ & 37 & 70 & 10 & 17 & 60 & 189 & 73 & 41 & 61 \\
\hline Influenza-Type $\mathrm{B}^{\mathrm{b}}$ & 1 & 3 & 0 & 3 & 3 & 28 & 6 & 0 & 3 \\
\hline Influenza-Type A \& B ${ }^{b}$ & 1 & 2 & 0 & 0 & 0 & 0 & 1 & 0 & 3 \\
\hline Influenza-Type NOSb & 1 & 0 & 0 & 0 & 1 & 0 & 1 & 3 & 99 \\
\hline Legionellosis & 3 & 5 & 0 & 0 & 0 & 5 & 4 & 4 & 3 \\
\hline Legionella longbeachae & 1 & 1 & 0 & 0 & 0 & 2 & 2 & 2 & 0 \\
\hline L.pneumophilab & 0 & 4 & 0 & 0 & 0 & 3 & 1 & 2 & 3 \\
\hline Legionnaire disease other & 2 & 0 & 0 & 0 & 0 & 0 & 1 & 0 & 0 \\
\hline Leprosy & 0 & 0 & 0 & 0 & 0 & 0 & 0 & 0 & 0 \\
\hline Leptospirosis $^{\mathrm{b}}$ & 0 & 0 & 0 & 1 & 0 & 1 & 1 & 2 & 3 \\
\hline Listeriosis $^{\mathrm{b}}$ & 0 & 0 & 1 & 0 & 0 & 5 & 0 & 0 & 0 \\
\hline Lymphogranuloma venereum (LGV) & 0 & 0 & 0 & 0 & 0 & 0 & 0 & 0 & 0 \\
\hline Malaria ${ }^{\mathrm{b}}$ & 3 & 6 & 0 & 0 & 1 & 14 & 2 & 4 & 2 \\
\hline Measles & 0 & 0 & 0 & 0 & 0 & 0 & 0 & 0 & 0 \\
\hline Measles laboratory confirmed & 0 & 0 & 0 & 0 & 0 & 0 & 0 & 0 & 0 \\
\hline Measles - other & 0 & 0 & 0 & 0 & 0 & 0 & 0 & 0 & 0 \\
\hline Meningococcal disease & 4 & 6 & 0 & 3 & 2 & 9 & 3 & 2 & 6 \\
\hline Meningococcal - serogroup $\mathrm{B}^{\mathrm{b}}$ & 2 & 5 & 0 & 3 & 2 & 6 & 2 & 2 & 3 \\
\hline Meningococcal - serogroup $C^{b}$ & 1 & 0 & 0 & 0 & 0 & 1 & 0 & 0 & 2 \\
\hline Meningococcal - serogroup W135 & 0 & 1 & 0 & 0 & 0 & 0 & 0 & 0 & 0 \\
\hline Meningococcal - serogroup $\mathrm{Y}^{\mathrm{b}}$ & 0 & 0 & 0 & 0 & 0 & 0 & 0 & 0 & 0 \\
\hline Meningococcal - other & 1 & 0 & 0 & 0 & 0 & 2 & 1 & 0 & 1 \\
\hline Mumps ${ }^{b}$ & 2 & 0 & 0 & 0 & 2 & 5 & 1 & 0 & 0 \\
\hline Pertussis & 64 & 55 & 6 & 57 & 18 & 200 & 64 & 48 & 92 \\
\hline Pneumococcal disease (invasive) ${ }^{b}$ & 21 & 14 & 7 & 15 & 12 & 64 & 17 & 26 & 20 \\
\hline Psittacosis ${ }^{b}$ & 4 & 1 & 1 & 2 & 3 & 5 & 0 & 1 & 2 \\
\hline Q fever ${ }^{b}$ & 4 & 13 & 3 & 46 & 8 & 21 & 57 & 16 & 27 \\
\hline Rubella & 0 & 0 & 0 & 3 & 0 & 0 & 1 & 0 & 0 \\
\hline Congenital rubellab & 0 & 0 & 0 & 0 & 0 & 0 & 0 & 0 & 0 \\
\hline Rubella - other ${ }^{b}$ & 0 & 0 & 0 & 3 & 0 & 0 & 1 & 0 & 0 \\
\hline Salmonella infection ${ }^{\mathrm{b}, \mathrm{d}}$ & 85 & 56 & 7 & 28 & 47 & 190 & 78 & 78 & 218 \\
\hline Shigellosis ${ }^{b}$ & 0 & 3 & 0 & 1 & 0 & 3 & 1 & 3 & 8 \\
\hline Syphilis & 9 & 8 & 14 & 16 & 16 & 25 & 8 & 27 & 12 \\
\hline Congenital syphilis & 0 & 0 & 0 & 0 & 1 & 0 & 0 & 0 & 0 \\
\hline Infectious syphilis $b, c$ & 1 & 2 & 1 & 0 & 3 & 12 & 2 & 2 & 5 \\
\hline Syphilis - otherb & 8 & 6 & 13 & 16 & 12 & 13 & 6 & 25 & 7 \\
\hline Tetanus & 0 & 0 & 0 & 0 & 0 & 0 & 0 & 0 & 1 \\
\hline Tuberculosis $^{\mathrm{b}}$ & 3 & 6 & 0 & 0 & 1 & 16 & 1 & 4 & 4 \\
\hline Typhoid $^{\mathrm{b}}$ & 1 & 0 & 0 & 0 & 0 & 0 & 0 & 0 & 0 \\
\hline Verotoxin-producing Escherichia coli infections ${ }^{b}$ & 3 & 1 & 0 & 0 & 0 & 9 & 4 & 0 & 1 \\
\hline
\end{tabular}

aYear of onset: the earlier of patient reported onset date, specimen date or date of notification. ' baboratory-confirmed cases only. Cincludes Syphilis primary, Syphilis secondary, Syphilis $<1$ year duration and Syphilis newly acquired. dincludes all paratyphoid cases. ${ }^{\mathrm{A}} \mathrm{AHS}$ further divided into the geographical region covered by their component public health unit. gRate is based on a denominator of 8000 persons. hIncludes cases with unknown PHU. NOS: not otherwise specified. No case of the following diseases have been notified since 1991: Plague $^{\mathrm{b}}$, Diphtheria ${ }^{\mathrm{b}}$, Granuloma inguinale ${ }^{\mathrm{b}}$, Lyssavirusb, Poliomyelitis ${ }^{\mathrm{b}}$, Rabies, Smallpox, Typhus ${ }^{\mathrm{b}}$, Viral haemorrhagic fever, Yellow fever. Due to data delay AIDS notifications will be reported in a later edition. 
Table 5. continued

\begin{tabular}{|c|c|c|c|c|c|c|c|c|c|c|}
\hline \multirow[t]{2}{*}{ Condition } & \multicolumn{2}{|c|}{ Northern Sydney Central Coast ${ }^{f}$} & \multicolumn{2}{|c|}{ South Eastern Sydney Illawara ${ }^{f}$} & \multicolumn{2}{|c|}{ Sydney South West ${ }^{f}$} & \multicolumn{2}{|c|}{ Sydney West ${ }^{t}$} & Justice & Total \\
\hline & Gosford & Hornsby & Wollongong & Randwick & Camperdown & Liverpool & Penrith & Parramatta & Health & \\
\hline Adverse event after immunisation & 15 & 14 & 19 & 25 & 6 & 16 & 19 & 28 & 0 & 224 \\
\hline Anthrax & 0 & 0 & 0 & 0 & 0 & 0 & 0 & 0 & 0 & 0 \\
\hline Arboviral infection & 67 & 40 & 100 & 43 & 18 & 14 & 14 & 24 & 1 & 1498 \\
\hline Barmah Forest virus ${ }^{b}$ & 16 & 6 & 69 & 4 & 3 & 2 & 2 & 6 & 1 & 573 \\
\hline Ross River virus ${ }^{\mathrm{b}}$ & 46 & 22 & 28 & 17 & 8 & 7 & 10 & 15 & 0 & 841 \\
\hline Other ${ }^{b}$ & 5 & 12 & 3 & 22 & 7 & 5 & 2 & 3 & 0 & 84 \\
\hline Blood lead level $\geq 15 \mathrm{ug} / \mathrm{dL}^{\mathrm{b}}$ & 3 & 10 & 21 & 13 & 14 & 23 & 8 & 30 & 0 & 263 \\
\hline Botulism & 0 & 0 & 0 & 0 & 0 & 0 & 0 & 0 & 0 & 0 \\
\hline Brucellosis $^{b}$ & 0 & 0 & 0 & 1 & 0 & 1 & 0 & 0 & 0 & 3 \\
\hline Chancroid $^{b}$ & 0 & 0 & 0 & 0 & 0 & 0 & 0 & 0 & 0 & 0 \\
\hline Chlamydia trachomatis infection & 579 & 1076 & 578 & 2283 & 1335 & 828 & 409 & 1042 & 95 & 12447 \\
\hline Congenital chlamydiab & 1 & 3 & 0 & 1 & 1 & 3 & 2 & 9 & 0 & 31 \\
\hline Chlamydia - other b & 578 & 1073 & 578 & 2282 & 1334 & 825 & 407 & 1033 & 95 & 12416 \\
\hline Cholera $^{\mathrm{b}}$ & 0 & 1 & 0 & 0 & 1 & 0 & 0 & 0 & 0 & 2 \\
\hline Creutzfeldt-Jakob disease $^{\mathrm{b}}$ & 1 & 0 & 2 & 1 & 0 & 1 & 1 & 0 & 0 & 7 \\
\hline Cryptosporidiosis $^{b}$ & 22 & 43 & 15 & 38 & 22 & 48 & 21 & 34 & 0 & 544 \\
\hline Gastroenteritis (institutional) & 431 & 1908 & 366 & 878 & 701 & 770 & 78 & 1552 & 12 & 10488 \\
\hline Giardiasis $^{b}$ & 81 & 313 & 83 & 331 & 201 & 119 & 85 & 267 & 2 & 1940 \\
\hline Gonorrhoeab $^{b}$ & 29 & 125 & 32 & 469 & 297 & 96 & 38 & 96 & 8 & 1384 \\
\hline Haemolytic uraemic syndrome & 0 & 0 & 1 & 1 & 1 & 1 & 0 & 1 & 0 & 13 \\
\hline Haemophilus influenzae serotype b & 0 & 1 & 0 & 1 & 0 & 1 & 0 & 1 & 0 & 7 \\
\hline Hib epiglottitis ${ }^{b}$ & 0 & 1 & 0 & 0 & 0 & 0 & 0 & 0 & 0 & 1 \\
\hline Hib meningitis ${ }^{b}$ & 0 & 0 & 0 & 0 & 0 & 0 & 0 & 0 & 0 & 2 \\
\hline Hib septicaemiab & 0 & 0 & 0 & 0 & 0 & 0 & 0 & 1 & 0 & 2 \\
\hline Hib infection NOS & 0 & 0 & 0 & 1 & 0 & 1 & 0 & 0 & 0 & 2 \\
\hline Hepatitis $A^{b}$ & 0 & 13 & 3 & 10 & 6 & 12 & 3 & 9 & 0 & 65 \\
\hline Hepatitis B & 33 & 286 & 46 & 386 & 427 & 600 & 39 & 557 & 44 & 2656 \\
\hline Hepatitis B - acute viral ${ }^{\mathrm{b}}$ & 2 & 6 & 0 & 14 & 3 & 12 & 1 & 1 & 1 & 56 \\
\hline Hepatitis B - other ${ }^{b}$ & 31 & 280 & 46 & 372 & 424 & 588 & 38 & 556 & 43 & 2600 \\
\hline Hepatitis C & 186 & 203 & 185 & 395 & 399 & 495 & 159 & 379 & 614 & 4259 \\
\hline Hepatitis C - acute viral ${ }^{b}$ & 0 & 0 & 0 & 2 & 21 & 5 & 0 & 1 & 4 & 53 \\
\hline Hepatitis C - other ${ }^{b}$ & 186 & 203 & 185 & 393 & 378 & 490 & 159 & 378 & 610 & 4206 \\
\hline Hepatitis $D^{b}$ & 1 & 0 & 2 & 3 & 0 & 0 & 1 & 4 & 0 & 11 \\
\hline Hepatitis $E^{b}$ & 1 & 1 & 0 & 3 & 3 & 0 & 0 & 0 & 0 & 8 \\
\hline HIV infection ${ }^{b}$ & 8 & 31 & 9 & 128 & 97 & 24 & 3 & 30 & 0 & 404 \\
\hline Influenza & 54 & 142 & 61 & 185 & 45 & 117 & 124 & 463 & 3 & 1918 \\
\hline Influenza-Type $A^{b}$ & 38 & 82 & 46 & 117 & 29 & 77 & 100 & 431 & 3 & 1487 \\
\hline Influenza-Type $\mathrm{B}^{\mathrm{b}}$ & 1 & 6 & 9 & 52 & 15 & 10 & 14 & 26 & 0 & 180 \\
\hline Influenza-Type A \& B b & 0 & 2 & 4 & 16 & 0 & 0 & 9 & 5 & 0 & 43 \\
\hline Influenza-Type NOSb & 15 & 52 & 2 & 0 & 1 & 30 & 1 & 1 & 0 & 208 \\
\hline Legionellosis & 4 & 8 & 6 & 13 & 8 & 13 & 10 & 19 & 0 & 105 \\
\hline Legionella longbeachae $e^{b}$ & 2 & 2 & 4 & 2 & 0 & 4 & 2 & 5 & 0 & 29 \\
\hline L.pneumophila ${ }^{b}$ & 2 & 6 & 2 & 11 & 8 & 9 & 8 & 14 & 0 & 73 \\
\hline Legionnaire disease other & 0 & 0 & 0 & 0 & 0 & 0 & 0 & 0 & 0 & 3 \\
\hline Leprosy & 0 & 0 & 0 & 0 & 0 & 0 & 0 & 4 & 0 & 4 \\
\hline Leptospirosis $^{b}$ & 0 & 0 & 0 & 0 & 0 & 0 & 0 & 0 & 0 & 8 \\
\hline Listeriosis $^{\mathrm{b}}$ & 0 & 3 & 2 & 5 & 1 & 2 & 1 & 2 & 0 & 22 \\
\hline Lymphogranuloma venereum (LGV) & 0 & 0 & 0 & 0 & 0 & 0 & 0 & 0 & 0 & 0 \\
\hline Malariab & 2 & 10 & 6 & 8 & 9 & 5 & 6 & 16 & 0 & 98 \\
\hline Measles & 0 & 1 & 0 & 1 & 0 & 1 & 0 & 0 & 0 & 4 \\
\hline Measles laboratory confirmed & 0 & 1 & 0 & 1 & 0 & 1 & 0 & 0 & 0 & 4 \\
\hline Measles - other & 0 & 0 & 0 & 0 & 0 & 0 & 0 & 0 & 0 & 0 \\
\hline Meningococcal disease & 6 & 13 & 4 & 17 & 5 & 12 & 5 & 14 & 0 & 112 \\
\hline Meningococcal - serogroup $\mathrm{B}^{\mathrm{b}}$ & 3 & 11 & 3 & 9 & 4 & 9 & 4 & 8 & 0 & 76 \\
\hline Meningococcal - serogroup $\mathrm{C}^{\mathrm{b}}$ & 1 & 0 & 1 & 3 & 1 & 0 & 0 & 0 & 0 & 10 \\
\hline Meningococcal - serogroup W135 & 0 & 0 & 0 & 0 & 0 & 1 & 0 & 0 & 0 & 2 \\
\hline Meningococcal - serogroup $\mathrm{Y}^{\mathrm{b}}$ & 2 & 1 & 0 & 1 & 0 & 0 & 0 & 1 & 0 & 5 \\
\hline Meningococcal - other & 0 & 1 & 0 & 4 & 0 & 2 & 1 & 5 & 0 & 19 \\
\hline Mumps $^{b}$ & 1 & 51 & 12 & 136 & 31 & 27 & 10 & 40 & 0 & 323 \\
\hline Pertussis & 76 & 281 & 78 & 319 & 177 & 153 & 87 & 317 & 0 & 2093 \\
\hline Pneumococcal disease (invasive) ${ }^{b}$ & 22 & 56 & 36 & 50 & 41 & 44 & 25 & 48 & 1 & 522 \\
\hline Psittacosis ${ }^{b}$ & 0 & 0 & 3 & 2 & 0 & 2 & 5 & 3 & 0 & 34 \\
\hline Q fever ${ }^{\mathrm{b}}$ & 3 & 1 & 12 & 1 & 0 & 0 & 1 & 2 & 0 & 215 \\
\hline Rubella & 0 & 1 & 0 & 0 & 1 & 0 & 0 & 3 & 0 & 9 \\
\hline Congenital rubella ${ }^{b}$ & 0 & 0 & 0 & 0 & 0 & 0 & 0 & 1 & 0 & 1 \\
\hline Rubella - other ${ }^{b}$ & 0 & 1 & 0 & 0 & 1 & 0 & 0 & 2 & 0 & 8 \\
\hline Salmonella infection ${ }^{\mathrm{b}, \mathrm{d}}$ & 142 & 339 & 86 & 295 & 239 & 251 & 102 & 306 & 1 & 2564 \\
\hline Shigellosis ${ }^{b}$ & 0 & 13 & 1 & 15 & 12 & 6 & 0 & 4 & 0 & 71 \\
\hline Syphilis & 21 & 54 & 31 & 333 & 251 & 138 & 27 & 95 & 19 & 1115 \\
\hline Congenital syphilis & 0 & 1 & 0 & 0 & 0 & 0 & 0 & 2 & 0 & 4 \\
\hline Infectious syphilis b,c & 1 & 17 & 7 & 238 & 92 & 11 & 4 & 28 & 2 & 434 \\
\hline Syphilis - other ${ }^{b}$ & 20 & 36 & 24 & 95 & 159 & 127 & 23 & 65 & 17 & 677 \\
\hline Tetanus & 0 & 0 & 1 & 0 & 0 & 0 & 0 & 0 & 0 & 2 \\
\hline Tuberculosis ${ }^{b}$ & 4 & 43 & 10 & 63 & 68 & 81 & 13 & 128 & 0 & 452 \\
\hline Typhoid & 0 & 2 & 0 & 2 & 3 & 4 & 0 & 13 & 0 & 26 \\
\hline $\begin{array}{l}\text { Verotoxin-producing } \\
\text { Escherichia coli infections }{ }^{b}\end{array}$ & 0 & 1 & 0 & 0 & 0 & 1 & 2 & 1 & 0 & 23 \\
\hline
\end{tabular}

aYear of onset: the earlier of patient reported onset date, specimen date or date of notification. ' baboratory-confirmed cases only. cincludes Syphilis primary, Syphilis secondary, Syphilis $<1$ year duration and Syphilis newly acquired. dincludes all paratyphoid cases. ${ }^{\mathrm{f}} \mathrm{AHS}$ further divided into the geographical region covered by their component public health unit. 9Rate is based on a denominator of 8000 persons. hIncludes cases with unknown PHU. NOS: not otherwise specified. No case of the following diseases have been notified since 1991: Plague $^{b}$, Diphtheria ${ }^{b}$, Granuloma inguinale ${ }^{b}$, Lyssavirusb, Poliomyelitis , Rabies, Smallpox, Typhus ${ }^{b}$, Viral haemorrhagic fever, Yellow fever.

Due to data delay AIDS notifications will be reported in a later edition. 
- Chlamydia trachomatis infections account for the most notifications in adults with rates peaking at 818 per 100000 in people aged between 16 and 24 years.

- Influenza is the most commonly reported notifiable disease in adults aged 65 years and older though this rate is markedly lower than that observed in children aged less than five years of age. Children and older adults are more likely to undergo testing for influenza.

\section{Outbreaks and threats}

Several notable disease outbreaks and threats were reported in 2007 in NSW. These included:

- an outbreak of Legionnaire disease in South East Sydney Illawarra AHS related to a contaminated cooling tower in Circular Quay in Sydney (January 2007)..$^{2}$

- hepatitis $\mathrm{C}$ transmission linked to a general medical practice in South East Sydney Illawarra Health Service that specialised in provision of vitamin and mineral injections (March 2007). ${ }^{3}$

- a sushi chef who was working while infectious with hepatitis A. Sydney South West Area Health Service provided immunoglobulin to over 400 people who had eaten potentially contaminated sushi. No subsequent hepatitis A cases were reported (March 2007). ${ }^{3}$

- a Salmonella infection outbreak associated with eating pork and chicken rolls from a bakery in Sydney South West Area Health Service (March 2007). ${ }^{3}$

\section{Conclusions}

Controlling the spread of sexually transmitted infections, in particular, remains a priority for NSW. This is evident in the re-emergence of infectious syphilis in the gay community and the high rates of Chlamydia trachomatis infections in young adults.

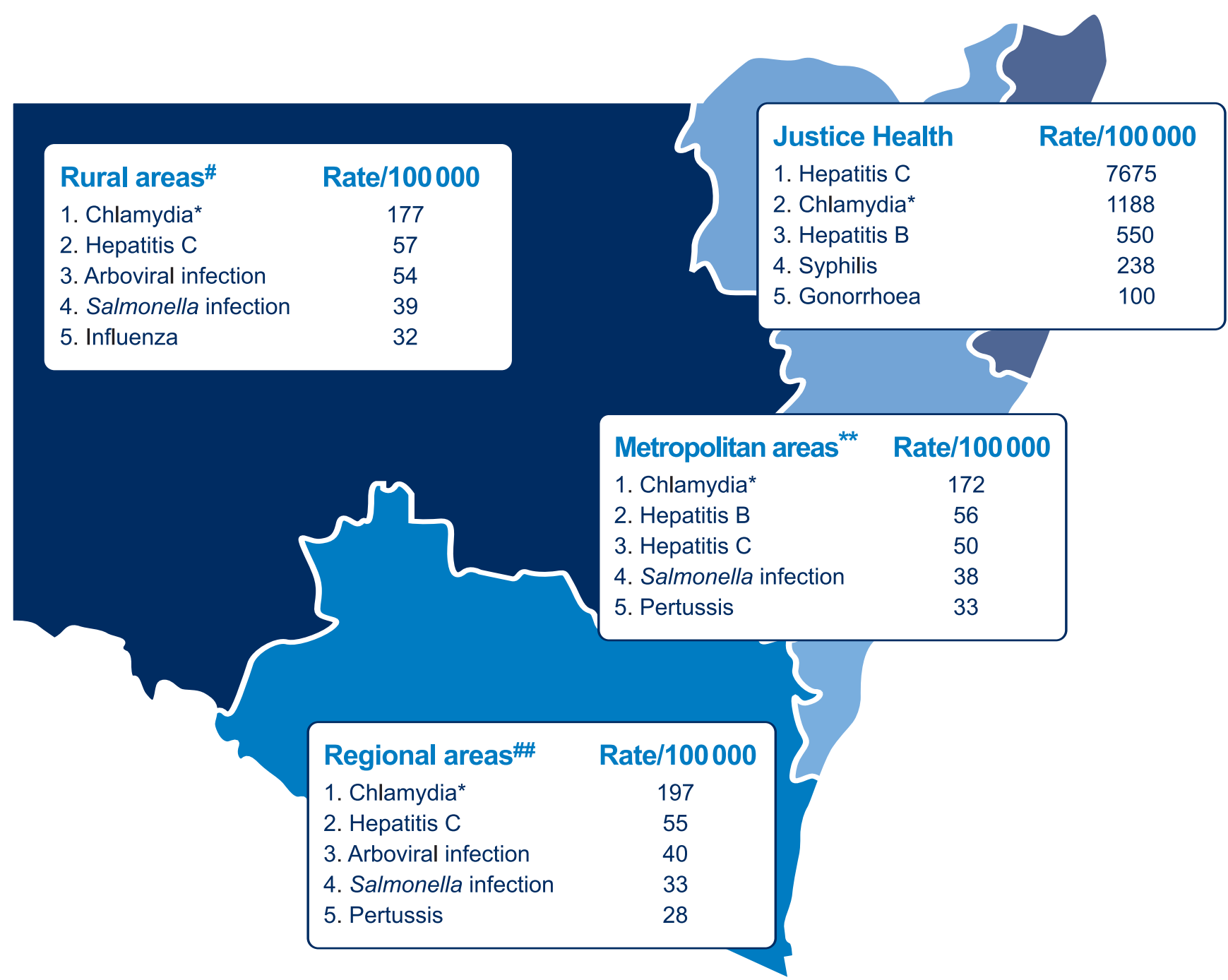

Figure 1. The five most commonly reported notifiable diseases by geographical area of residence at the time of notification in NSW, 2007. \# Includes Greater Southern, Greater Western, Hunter New England (Tamworth region) and North Coast Area Health Services. \#\# Includes Northern Sydney Central Coast (Gosford region), South East Sydney Illawarra (Wollongong region) and Hunter New England (Newcastle region). *Refers to notifications of Chlamydia trachomatis. ** Includes Northern Sydney Central Coast (Hornsby region), South East Sydney Illawarra (Randwick region), Sydney South West and Sydney West Area Health Services. Source: NSW Notifiable Diseases Database. 
Table 6. Disease notifications by age group and sex of the case, NSW, 2006

\begin{tabular}{|c|c|c|c|c|c|c|c|c|c|c|c|c|c|}
\hline \multirow[t]{2}{*}{ Condition } & \multicolumn{2}{|c|}{$0-4$ years } & \multicolumn{2}{|c|}{$5-24$ years } & \multicolumn{2}{|c|}{ 25-44 years } & $45-6$ & rears & $65+$ & ears & & & Totale \\
\hline & $\mathbf{F}$ & M & $F$ & M & $\mathbf{F}$ & M & $\mathbf{F}$ & M & $\mathbf{F}$ & M & $\mathbf{F}$ & M & \\
\hline Adverse event after immunisation & 15 & 18 & 133 & 5 & 21 & 2 & 16 & 2 & 6 & 6 & 191 & 33 & 224 \\
\hline Anthrax & 0 & 0 & 0 & 0 & 0 & 0 & 0 & 0 & 0 & 0 & 0 & 0 & 0 \\
\hline Arboviral infection & 6 & 1 & 108 & 74 & 285 & 242 & 283 & 300 & 88 & 109 & 770 & 726 & 1498 \\
\hline Barmah Forest virus ${ }^{b}$ & 3 & 0 & 35 & 24 & 91 & 91 & 130 & 134 & 30 & 33 & 289 & 282 & 573 \\
\hline Ross River virus ${ }^{\mathrm{b}}$ & 3 & 1 & 64 & 44 & 175 & 132 & 143 & 150 & 56 & 73 & 441 & 400 & 841 \\
\hline Other ${ }^{b}$ & 0 & 0 & 9 & 6 & 19 & 19 & 10 & 16 & 2 & 3 & 40 & 44 & 84 \\
\hline Blood lead level $\geq 15 \mathrm{ug} / \mathrm{dL}^{\mathrm{b}}$ & 0 & 9 & 3 & 46 & 7 & 109 & 3 & 71 & 1 & 14 & 14 & 249 & 263 \\
\hline Botulism & & & & & & & & & & & & & 0 \\
\hline Brucellosis $^{b}$ & 0 & 0 & 0 & 2 & 0 & 0 & 0 & 0 & 0 & 1 & 0 & 3 & 3 \\
\hline Chancroid $^{\mathrm{b}}$ & & & & & & & & & & & & & 0 \\
\hline Chlamydia trachomatis infection & 22 & 18 & 4783 & 2299 & 2172 & 2571 & 111 & 400 & 9 & 24 & 7097 & 5313 & 12447 \\
\hline Congenital chlamydiab & 14 & 13 & 3 & 0 & 0 & 0 & 0 & 0 & 0 & 0 & 18 & 13 & 31 \\
\hline Chlamydia - other b & 8 & 5 & 4780 & 2299 & 2172 & 2571 & 111 & 400 & 9 & 24 & 7079 & 5300 & 12416 \\
\hline Cholera ${ }^{b}$ & 0 & 0 & 0 & 0 & 0 & 1 & 0 & 1 & 0 & 0 & 0 & 2 & 2 \\
\hline Creutzfeldt-Jakob disease $^{b}$ & 0 & 0 & 0 & 0 & 0 & 0 & 3 & 1 & 2 & 1 & 5 & 2 & 7 \\
\hline Cryptosporidiosis $^{b}$ & 80 & 108 & 78 & 122 & 61 & 50 & 19 & 17 & 4 & 4 & 242 & 301 & 544 \\
\hline Giardiasis $^{b}$ & 227 & 329 & 159 & 202 & 375 & 259 & 137 & 121 & 74 & 51 & 973 & 963 & 1940 \\
\hline Gonorrhoeab $^{\mathrm{b}}$ & 0 & 0 & 102 & 245 & 84 & 731 & 19 & 195 & 1 & 4 & 206 & 1175 & 1384 \\
\hline Haemolytic uraemic syndrome & 2 & 3 & 1 & 2 & 2 & 0 & 1 & 1 & 1 & 0 & 7 & 6 & 13 \\
\hline Haemophilus influenzae serotype b & 3 & 1 & 0 & 1 & 0 & 0 & 1 & 1 & 0 & 0 & 4 & 3 & 7 \\
\hline Hib epiglottitis ${ }^{b}$ & 0 & 0 & 0 & 0 & 0 & 0 & 0 & 1 & 0 & 0 & 0 & 1 & 1 \\
\hline Hib meningitis ${ }^{b}$ & 2 & 0 & 0 & 0 & 0 & 0 & 0 & 0 & 0 & 0 & 2 & 0 & 2 \\
\hline Hib septicaemiab & 0 & 1 & 0 & 0 & 0 & 0 & 1 & 0 & 0 & 0 & 1 & 1 & 2 \\
\hline Hib infection NOSb & 1 & 0 & 0 & 1 & 0 & 0 & 0 & 0 & 0 & 0 & 1 & 1 & 2 \\
\hline Hepatitis $A^{b}$ & 4 & 3 & 7 & 12 & 11 & 15 & 4 & 5 & 2 & 2 & 28 & 37 & 65 \\
\hline Hepatitis B & 6 & 7 & 232 & 215 & 694 & 748 & 242 & 384 & 45 & 64 & 1219 & 1418 & 2656 \\
\hline Hepatitis B - acute viral ${ }^{b}$ & 1 & 0 & 8 & 5 & 15 & 13 & 2 & 9 & 1 & 1 & 27 & 28 & 56 \\
\hline Hepatitis B - other ${ }^{b}$ & 5 & 7 & 224 & 210 & 679 & 735 & 240 & 375 & 44 & 63 & 1192 & 1390 & 2600 \\
\hline Hepatitis C & 9 & 15 & 229 & 243 & 876 & 1543 & 368 & 820 & 60 & 71 & 1543 & 2692 & 4259 \\
\hline Hepatitis C - acute viral ${ }^{b}$ & 1 & 1 & 10 & 6 & 11 & 18 & 2 & 4 & 0 & 0 & 24 & 29 & 53 \\
\hline Hepatitis C - other ${ }^{b}$ & 8 & 14 & 219 & 237 & 865 & 1525 & 366 & 816 & 60 & 71 & 1519 & 2663 & 4206 \\
\hline Hepatitis $D^{b}$ & 0 & 0 & 0 & 0 & 1 & 6 & 1 & 3 & 0 & 0 & 2 & 9 & 11 \\
\hline Hepatitis $\mathrm{E}^{\mathrm{b}}$ & 0 & 0 & 1 & 4 & 0 & 3 & 0 & 0 & 0 & 0 & 1 & 7 & 8 \\
\hline HIV infection ${ }^{b}$ & 0 & 0 & 3 & 21 & 34 & 240 & 10 & 89 & 2 & 3 & 49 & 353 & 404 \\
\hline Influenza & 174 & 244 & 180 & 200 & 199 & 197 & 203 & 180 & 167 & 170 & 923 & 991 & 1918 \\
\hline Influenza-Type $A^{b}$ & 141 & 202 & 138 & 160 & 161 & 149 & 149 & 134 & 129 & 122 & 718 & 767 & 1487 \\
\hline Influenza-Type $\mathrm{B}^{\mathrm{b}}$ & 10 & 18 & 17 & 14 & 13 & 27 & 21 & 19 & 18 & 22 & 79 & 100 & 180 \\
\hline Influenza-Type A \& B b & 0 & 0 & 5 & 6 & 3 & 3 & 6 & 7 & 4 & 9 & 18 & 25 & 43 \\
\hline Influenza-Type NOS & 23 & 24 & 20 & 20 & 22 & 18 & 27 & 20 & 16 & 17 & 108 & 99 & 208 \\
\hline Legionellosis & 0 & 0 & 1 & 0 & 4 & 11 & 19 & 30 & 9 & 30 & 33 & 71 & 105 \\
\hline Legionella longbeachae $e^{b}$ & 0 & 0 & 1 & 0 & 1 & 3 & 8 & 5 & 2 & 9 & 12 & 17 & 29 \\
\hline L.pneumophilab & 0 & 0 & 0 & 0 & 3 & 8 & 10 & 24 & 6 & 21 & 19 & 53 & 73 \\
\hline Legionnaire disease other & 0 & 0 & 0 & 0 & 0 & 0 & 1 & 1 & 1 & 0 & 2 & 1 & 3 \\
\hline Leprosy & 0 & 0 & 0 & 0 & 1 & 0 & 1 & 1 & 0 & 1 & 2 & 2 & 4 \\
\hline Leptospirosis $^{b}$ & 0 & 0 & 1 & 1 & 0 & 3 & 1 & 1 & 0 & 1 & 2 & 6 & 8 \\
\hline Listeriosis $^{\mathrm{b}}$ & 1 & 0 & 0 & 0 & 2 & 1 & 0 & 2 & 8 & 8 & 11 & 11 & 22 \\
\hline Lymphogranuloma venereum (LGV) & & & & & & & & & & & & & 0 \\
\hline Malariab & 0 & 1 & 0 & 1 & 1 & 1 & 0 & 0 & 0 & 0 & 1 & 3 & 98 \\
\hline Measles & 0 & 1 & 6 & 24 & 14 & 29 & 2 & 18 & 1 & 2 & 23 & 74 & 4 \\
\hline $\begin{array}{l}\text { Measles laboratory confirmed } \\
\text { Measles - other }\end{array}$ & 0 & 1 & 0 & 1 & 1 & 1 & 0 & 0 & 0 & 0 & 1 & 3 & $\begin{array}{l}4 \\
0\end{array}$ \\
\hline Meningococcal disease & 18 & 25 & 21 & 14 & 4 & 10 & 8 & 4 & 4 & 4 & 55 & 57 & 112 \\
\hline Meningococcal - serogroup $\mathrm{B}^{\mathrm{b}}$ & 15 & 18 & 12 & 10 & 2 & 9 & 4 & 3 & 3 & 0 & 36 & 40 & 76 \\
\hline Meningococcal - serogroup $\mathrm{C}^{\mathrm{b}}$ & 0 & 1 & 4 & 0 & 1 & 1 & 2 & 1 & 0 & 0 & 7 & 3 & 10 \\
\hline Meningococcal - serogroup W135b & 0 & 1 & 0 & 0 & 0 & 0 & 1 & 0 & 0 & 0 & 1 & 1 & 2 \\
\hline Meningococcal - serogroup $\mathrm{Y}^{\mathrm{b}}$ & 0 & 0 & 1 & 0 & 0 & 0 & 0 & 0 & 1 & 3 & 2 & 3 & 5 \\
\hline Meningococcal - other & 3 & 5 & 4 & 4 & 1 & 0 & 1 & 0 & 0 & 1 & 9 & 10 & 19 \\
\hline Mumps ${ }^{b}$ & 2 & 3 & 26 & 64 & 83 & 116 & 13 & 14 & 1 & 0 & 125 & 197 & 323 \\
\hline Pertussis & 100 & 74 & 217 & 165 & 346 & 210 & 400 & 259 & 176 & 134 & 1239 & 842 & 2093 \\
\hline Pneumococcal disease (invasive) ${ }^{b}$ & 40 & 43 & 16 & 24 & 38 & 54 & 55 & 72 & 82 & 98 & 231 & 291 & 522 \\
\hline Psittacosis ${ }^{b}$ & 0 & 0 & 1 & 0 & 2 & 1 & 6 & 15 & 1 & 7 & 10 & 23 & 34 \\
\hline Q fever ${ }^{\mathrm{b}}$ & 1 & 0 & 12 & 21 & 25 & 49 & 23 & 64 & 9 & 11 & 70 & 145 & 215 \\
\hline Rubella & 1 & 2 & 0 & 0 & 4 & 1 & 0 & 0 & 0 & 0 & 5 & 3 & 9 \\
\hline Congenital rubellab & 0 & 0 & 0 & 0 & 0 & 0 & 0 & 0 & 0 & 0 & 0 & 0 & 1 \\
\hline Rubella - other ${ }^{b}$ & 1 & 2 & 0 & 0 & 4 & 1 & 0 & 0 & 0 & 0 & 5 & 3 & 8 \\
\hline Salmonella infection ${ }^{\mathrm{b}, \mathrm{d}}$ & 310 & 317 & 328 & 370 & 264 & 290 & 208 & 236 & 128 & 99 & 1238 & 1313 & 2564 \\
\hline Shigellosis ${ }^{b}$ & 1 & 2 & 9 & 4 & 11 & 21 & 9 & 9 & 3 & 2 & 33 & 38 & 71 \\
\hline Syphilis & 1 & 4 & 14 & 42 & 131 & 455 & 52 & 266 & 49 & 97 & 247 & 864 & 1115 \\
\hline Congenital syphilis & 1 & 2 & 0 & 0 & 0 & 0 & 0 & 0 & 0 & 0 & 1 & 2 & 4 \\
\hline Infectious syphilis b,c & 0 & 0 & 3 & 21 & 17 & 274 & 5 & 106 & 1 & 7 & 26 & 408 & 434 \\
\hline Syphilis - other & 0 & 2 & 11 & 21 & 114 & 181 & 47 & 160 & 48 & 90 & 220 & 454 & 677 \\
\hline Tetanus & 0 & 0 & 0 & 0 & 0 & 0 & 0 & 0 & 1 & 1 & 1 & 1 & 2 \\
\hline Tuberculosis $^{b}$ & 4 & 4 & 37 & 45 & 76 & 95 & 54 & 60 & 33 & 41 & 204 & 245 & 452 \\
\hline Typhoid $^{b}$ & 3 & 1 & 7 & 4 & 7 & 2 & 1 & 0 & 0 & 1 & 18 & 8 & 26 \\
\hline $\begin{array}{l}\text { Verotoxin-producing } \\
\text { Escherichia coli infections }{ }^{\text {b }}\end{array}$ & 0 & 2 & 1 & 2 & 4 & 3 & 3 & 0 & 3 & 5 & 11 & 12 & 23 \\
\hline $\begin{array}{l}\text { aYear of onset: the earlier of patient reported } \\
\text { cincludes Syphilis primary, Syphilis secondary } \\
\text { encludes cases with unknown-age and sex a } \\
\text { Due to data delay AIDS notifications will be }\end{array}$ & $\begin{array}{l}\text { Who } \\
\text { a lat }\end{array}$ & $\begin{array}{l}\text { tify as } \\
\text { ition. }\end{array}$ & en & s: & $\mathrm{ab}$ & III & $\mathrm{dca}$ & $\begin{array}{l}\text { y. } \\
\text { ases. }\end{array}$ & & & & & \\
\hline
\end{tabular}


While transmission of some vaccine preventable diseases has been limited in NSW, the challenge still remains to increase vaccination rates among adolescents and young adults to reduce their susceptibility to diseases such as mumps, measles and pertussis.

The increase in Salmonella infections serves as a timely reminder to all to ensure thorough cooking and safe handling of high-risk foods such as raw chicken and other meats, and undercooked, cracked or soiled eggs, while the Legionnaire disease outbreak highlights the importance of cooling tower maintenance.

We thank all those general and specialist medical practices, laboratories, hospitals, schools, child-care centres and others who have notified diseases of public health significance to their local public health units for investigation and control.

\section{References}

1. OzFoodNet, 4th Quarterly and Annual Reports 2007. Accessed at http://www.health.nsw.gov.au/publichealth/infectious.

2. NSW Department of Health Communicable Diseases Report, NSW, for January and February 2007. N S W Public Health Bull 2007; 18(3-4): 66-8.

3. NSW Department of Health Communicable Diseases Report, NSW, for March and April 2007. N S W Public Health Bull 2007; 18(5-6): 100-3.

Erratum. The following correction should be made in Table 5 of the 2005 Annual Report (N SW Public Health Bull 2006; 17(5-6): 74): the headings 'Male' and 'Female' should be interchanged on each column. 